\title{
On Bernoulli identities and applications
}

Minking Eie and King F. Lai

\section{Part I}

Abstract. Bernoulli numbers appear as special values of zeta functions at integers and identities relating the Bernoulli numbers follow as a consequence of properties of the corresponding zeta functions. The most famous example is that of the special values of the Riemann zeta function and the Bernoulli identities due to Euler. In this paper we introduce a general principle for producing Bernoulli identities and apply it to zeta functions considered by Shintani, Zagier and Eie. Our results include some of the classical results of Euler and Ramanujan. Kummer's congruences play important roles in the investigation of $p$ adic interpolation of the classical Riemann zeta function. It asserts congruence relations among Bernoulli numbers, i.e.

$$
\left(1-p^{m-1}\right) \frac{B_{m}}{m} \equiv\left(1-p^{n-1}\right) \frac{B_{n}}{n} \quad\left(\bmod p^{N+1}\right)
$$

if $m \equiv n\left(\bmod (p-1) p^{N}\right)$ and $(p-1)$ is not a divisor of $m$. In the second part of this paper, we use a simple Bernoulli identity to prove that

$$
\begin{aligned}
& \left(1-p^{m-1}\right) \frac{B_{m}}{m} \\
& \equiv \frac{p^{-(N+1)}}{m} \sum_{\substack{(j, p)=1 \\
1 \leq j<p^{N+1}}} j^{m}-\frac{1}{2} \sum_{\substack{(j, p)=1 \\
1 \leq j<p^{N+1}}} j^{m-1}\left(\bmod p^{N+1}\right) .
\end{aligned}
$$


We then deduce from this Kummer's congruence by using von Staudt's theorem and Euler's generalization of Fermat's theorem

$$
a^{m} \equiv a^{n} \quad\left(\bmod p^{N+1}\right),
$$

if $a$ is relative prime to $p$ and $m \equiv n\left(\bmod (p-1) p^{N}\right)$. Our argument can be applied to derive congruences among Bernoulli polynomials and in general the special values at negative integers of zeta functions associated with rational functions considered by Eie.

\section{Introduction.}

Let $m_{1}, \ldots, m_{r}$ be positive integers and $P(T)$ be a polynomial in $T$ with complex coefficients of degree less than $m_{1}+\cdots+m_{r}$. For $|T|<1$, we let

$$
F(T)=\frac{P(T)}{\left(1-T^{m_{1}}\right) \cdots\left(1-T^{m_{r}}\right)}=\sum_{k=0}^{\infty} a(k) T^{k} .
$$

Such functions occur as generating functions of partition numbers ( $c f$. Hardy and Wright [5, Chapter XIX]) and dimensions of spaces of automorphic forms - e.g. if we let $a(k)$ be the dimension of the space of Siegel modular forms of genus 2 and weight $k$, then

$$
\sum_{k=0}^{\infty} a(k) T^{k}=\frac{1+T^{35}}{\left(1-T^{4}\right)\left(1-T^{6}\right)\left(1-T^{10}\right)\left(1-T^{12}\right)}
$$

(cf. Igusa [6]). The value of $a(k)$ is determined by $F$ via the residue theorem as

$$
a(k)=\frac{1}{2 \pi i} \int_{\mathcal{C}} \frac{F(z) d z}{z^{k+1}},
$$

where $\mathcal{C}$ is a sufficiently small circle centered at the origin going counterclockwise.

The generating function of the numbers $a(k)$ is the Dirichlet series

$$
Z_{F}(s)=\sum_{k=1}^{\infty} a(k) k^{-s}
$$

( $c f$. Hardy and Wright [5, Chapter XVII]). This zeta function is related to $F(T)$ via a Mellin transform

$$
Z_{F}(s) \Gamma(s)=\int_{0}^{\infty} t^{s-1}\left(F\left(e^{-t}\right)-F(0)\right) d t,
$$


for Re $s$ sufficiently large. Our underlying principle is to evaluate $F(T)$ in two ways, yielding a Bernoulli identity, with special values of the zeta functions of Shintani [8], Zagier [9] and Eie [2], [3] on the one hand, the special values of classical zeta functions of Riemann and Hurwitz and sums of residues on the other. One gets easily this way Euler's identity: if $n \geq 2$,

$$
\sum_{k=1}^{n-1} \frac{(2 n) !}{(2 k) !(2 n-2 k) !} B_{2 k} B_{2 n-2 k}=-(2 n+1) B_{2 n},
$$

(cf. [1, Part I, p. 122]) and Ramanujan's identities $(\alpha, \beta>0$ with $\left.\alpha \beta=\pi^{2}\right)$,

1) if $n>1$,

$$
\alpha^{n} \sum_{k=1}^{\infty} \frac{k^{2 n-1}}{e^{2 \alpha k}-1}-(-\beta)^{n} \sum_{k=1}^{\infty} \frac{k^{2 n-1}}{e^{2 \beta k}-1}=\left(\alpha^{n}-(-\beta)^{n}\right) \frac{B_{2 n}}{4 n},
$$

2) if $n \in \mathbb{Z}$,

$$
\begin{aligned}
& \alpha^{-n}\left(\frac{1}{2} \zeta(2 n+1)+\sum_{k=1}^{\infty} \frac{k^{-2 n-1}}{e^{2 \alpha k}-1}\right) \\
&-(-\beta)^{-n}\left(\frac{1}{2} \zeta(2 n+1)+\sum_{k=1}^{\infty} \frac{k^{-2 n-1}}{e^{2 \beta k}-1}\right) \\
&=-2^{2 n} \sum_{k=0}^{n+1}(-1)^{k} \frac{B_{2 k}}{(2 k) !} \frac{B_{2 n+2-2 k}}{(2 n+2-2 k) !} \alpha^{n+1-k} \beta^{k},
\end{aligned}
$$

3) if $n \geq 1$,

$$
\begin{gathered}
\alpha^{-n} \sum_{k=1}^{\infty}(-1)^{k+1} \frac{\operatorname{csch}(\alpha k)}{k^{2 n+1}}-(-\beta)^{-n} \sum_{k=1}^{\infty}(-1)^{k+1} \frac{\operatorname{csch}(\beta k)}{k^{2 n+1}} \\
=2^{2 n+1} \sum_{k=0}^{n+1}(-1)^{k} \frac{B_{2 k}\left(\frac{1}{2}\right)}{(2 k) !} \frac{B_{2 n+2-2 k}\left(\frac{1}{2}\right)}{(2 n+2-2 k) !} \alpha^{n+1-k} \beta^{k}
\end{gathered}
$$

(cf. [1, Part II, Chapter 14]).

In the first part of this paper we present some new Bernoulli identities. In view of the current motivic interest in special values of zeta 
functions, one cannot help from wondering if there is an abstract framework giving a unified explanation of these identities as in the case of polylogarithms (cf. Zagier [10]).

In the second part of the paper the Bernoulli identities are used to give new proofs of classical Kummer congruences. The Bernoulli numbers $B_{n}(n=0,1,2, \ldots)$ and Bernoulli polynomials $B_{n}(x)(n=$ $0,1,2, \ldots)$ are defined by

$$
\frac{t}{e^{t}-1}=\sum_{n=0}^{\infty} \frac{B_{n} t^{n}}{n !}, \quad|t|<2 \pi,
$$

and

$$
\frac{t e^{x t}}{e^{t}-1}=\sum_{n=0}^{\infty} \frac{B_{n}(x) t^{n}}{n !}, \quad|t|<2 \pi .
$$

Suppose that $m, n$ are positive even integers, $p$ is an odd prime with $p-1$ not a divisor of $m$ and $N$ is a non-negative integer. Kummer's congruences asserted that if

$$
m \equiv n \quad\left(\bmod (p-1) p^{N}\right)
$$

then

$$
\left(1-p^{m-1}\right) \frac{B_{m}}{m} \equiv\left(1-p^{n-1}\right) \frac{B_{n}}{n} \quad\left(\bmod p^{N+1}\right) .
$$

Kummer's congruences play important roles in the $p$-adic interpolation of the classical Riemman zeta function. Indeed if we consider the function

$$
\zeta_{p}(s)=\left(1-p^{-s}\right) \zeta(s)=\sum_{\substack{n=1 \\(n, p)=1}} n^{-s}, \quad \operatorname{Re} s>1 .
$$

Then the congruences tell us that $\zeta_{p}(s)$ is a continuous function on the ring of $p$-adic integers $\mathbb{Z}_{p}$, i.e.,

$$
\zeta_{p}(1-m) \equiv \zeta_{p}(1-n) \quad\left(\bmod p^{N+1}\right),
$$

if $m \equiv n\left(\bmod (p-1) p^{N}\right)$.

One can construct a $p$-adic measure $\mu$ on $\mathbb{Z}_{p}$ and express $\zeta_{p}(1-m)$ as a constant multiple of the $p$-adic integration

$$
\int x^{m-1} d \mu(x)
$$


where the integration is over $\mathbb{Z}_{p}^{*}$ (see for example Koblitz [9]). Note that for $x \in\left(\mathbb{Z} / p^{N+1} \mathbb{Z}\right)^{*}$, the set of invertible elements of the quotent $\operatorname{ring} \mathbb{Z} / p^{N+1} \mathbb{Z}$, one has

$$
x^{m-1} \equiv x^{n-1} \quad\left(\bmod p^{N+1}\right),
$$

if

$$
m \equiv n \quad\left(\bmod (p-1) p^{N}\right) .
$$

So that Kummer's congruences follow as easy consequences by a simple argument (cf. [6]).

Here we shall develop another elementary proof of Kummer's congruences by a simple identity among Riemann zeta function and Hurwitz zeta functions,

$$
\left(1-p^{-s}\right) \zeta(s)=p^{-(N+1) s} \sum_{\substack{(j, p)=1 \\ 1 \leq j<p^{N+1}}} \zeta\left(s ; \frac{j}{p^{N+1}}\right),
$$

where the Hurwitz zeta function is defined as

$$
\zeta(s ; \delta)=\sum_{n=0}^{\infty}(n+\delta)^{-s}, \quad \operatorname{Re} s>1, \delta>0 .
$$

Such an identity follows easily from the consideration of zeta functions associated with rational functions of the form

$$
F(T)=\frac{P(T)}{\left(1-T^{m_{1}}\right) \cdots\left(1-T^{m_{r}}\right)}
$$

(see Part I).

Note that both the Riemann zeta function $\zeta(s)$ and Hurwitz zeta function $\zeta(s ; \delta)$ have analytic continuations in the whole complex plane. Moreover, their special values at non-positive integers are given by Bernoulli numbers and Bernoulli polynomials, respectively. Specifically, one has

$$
\zeta(1-m)=(-1)^{m-1} \frac{B_{m}}{m} \quad \text { and } \quad \zeta(1-m ; \delta)=-\frac{B_{m}(\delta)}{m} .
$$

Set $s=1-m$ in the identity (I), we get

$$
\left(1-p^{m-1}\right) \frac{B_{m}}{m}=\frac{1}{m} \sum_{\substack{(j, p)=1 \\
1 \leq j<p^{N+1}}} \sum_{l=0}^{m}\left(\begin{array}{c}
m \\
l
\end{array}\right) B_{l} j^{m-l} p^{(N+1)(l-1)} .
$$


Here

$$
\left(\begin{array}{c}
m \\
l
\end{array}\right)=\frac{m !}{l !(m-l) !}
$$

is the binomial coefficient.

On the other hand, von Staudt's theorem ([2, Chapter 5, Theorem 4]) implies that $p B_{l}$ is alway $p$-integral, i.e. it contains no divisor of $p$ in the denominator of $p B_{l}$. So after modulo $p^{N+1}$, we get

$$
\begin{aligned}
\left(1-p^{m-1}\right) \frac{B_{m}}{m} \equiv & \frac{1}{m} \sum_{\substack{(j, p)=1 \\
1 \leq j<p^{N+1}}} j^{m} p^{-(N+1)} \\
& -\frac{1}{2} \sum_{\substack{(j, p)=1 \\
1 \leq j<p^{N+1}}} j^{m-1}\left(\bmod p^{N+1}\right) .
\end{aligned}
$$

Next we evaluate the sum

$$
\sum_{\substack{(j, p)=1 \\ 1 \leq j<p^{N+1}}} j^{m},
$$

in the multiplicative group $\left(\mathbb{Z} / p^{N+1} \mathbb{Z}\right)^{*}$ by decomposing it into a direct product of finite cyclic groups and we obtain Kummer's congruences by assuming von Staudt's Theorem; finally we give a proof of von Staudt's theorem by using the Bernoulli identity (II) with $N=0$.

At the end of the paper we extend Kummer's congruences on Bernoulli numbers to congruences on Bernoulli polynomials.

\section{Special values of zeta functions.}

\subsection{Bernoulli numbers and Bernoulli polynomials.}

We recall some results on special values of zeta functions.

For the Riemann zeta function

$$
\zeta(s)=\sum_{n=1}^{\infty} n^{-s}, \quad \operatorname{Re} s>1
$$


and the Hurwitz zeta function

$$
\zeta(s ; \delta)=\sum_{n=0}^{\infty}(n+\delta)^{-s}, \quad \delta>0, \operatorname{Re} s>1,
$$

it is well known that for an integer $m \geq 0$,

$$
\zeta(-m)=(-1)^{m} \frac{B_{m+1}}{m+1} \quad \text { and } \quad \zeta(-m ; \delta)=-\frac{B_{m+1}(\delta)}{m+1} .
$$

\subsection{Zeta functions associated with linear forms.}

Let $\beta=\left(\beta_{1}, \ldots, \beta_{r}\right)$ be an $r$-tuple of nonnegative integers and $L(x)=a_{1} x_{1}+\cdots+a_{r} x_{r}+\delta$ be a linear form with

$$
\operatorname{Re} a_{j}>0 \quad \text { and } \quad \operatorname{Re}\left(\delta+\sum_{j=1}^{r} a_{j}\right)>0 .
$$

For $\operatorname{Re} s>r+|\beta|$, define the zeta function associated with $L$ as

$$
\begin{aligned}
Z(L, \beta, s) & =\sum_{n \in \mathbb{N}^{r}} n^{\beta} L(n)^{-s} \\
& =\sum_{n_{1}=1}^{\infty} \cdots \sum_{n_{r}=1}^{\infty} n_{1}^{\beta_{1}} \cdots n_{r}^{\beta_{r}}\left(a_{1} n_{1}+\cdots+a_{r} n_{r}+\delta\right)^{-s}
\end{aligned}
$$

where we use the notation $n^{\beta}=n_{1}^{\beta_{1}} \cdots n_{r}^{\beta_{r}}$.

These zeta functions were first considered in more general context by Eie in [2]. In particular, they have meromorphic continuations in the whole complex $s$-plane. Furthermore, their special values at nonpositive integers are given explicitly there. Here we summarize the results we need from [3].

For any polynomial $f(x)$ of $p$ variables and degree $k$

$$
f(x)=\sum_{|\alpha|=0}^{k} a_{\alpha} x_{1}^{\alpha_{1}} \cdots x_{p}^{\alpha_{p}}
$$

we let

$$
J^{p}(f(x))=\sum_{|\alpha|=0}^{k} a_{\alpha} \zeta\left(-\alpha_{1}\right) \cdots \zeta\left(-\alpha_{p}\right)=\sum_{|\alpha|=0}^{k} a_{\alpha} \prod_{j=1}^{p} \frac{(-1)^{\alpha_{j}} B_{\alpha_{j}+1}}{\alpha_{j}+1}
$$


where $\alpha=\left(\alpha_{1}, \ldots, \alpha_{p}\right)$ ranges over all $p$-tuples of non-negative integers and $|\alpha|=\alpha_{1}+\cdots+\alpha_{p}$.

Also for any nonempty subset $S$ of the index set $I=\{1,2, \ldots, r\}$, we let

$$
L_{S}(x)=\sum_{i \in I-S} a_{i} x_{i}+\delta=L(x)-\sum_{j \in S} a_{j} x_{j}
$$

and $|S|$ be the cardinal number of $S$.

The following proposition is an immediate consequence of the main theorem in [3].

Proposition 1. For any integer $m \geq 0$, the special value at $s=-m$ of $Z(L, \beta ; s)$ is given by

$$
\begin{aligned}
Z(L, \beta ;-m)= & J^{r}\left(x^{\beta} L^{m}(x)\right) \\
+ & +\sum_{S}\left(\prod_{j \in S} \frac{(-1)^{\beta_{j}+1} \beta_{j} !}{a_{j}^{\beta_{j}+1}}\right) \frac{1}{\alpha(S) !} J^{r-|S|} \\
& \cdot\left(\prod_{i \notin S} x_{i}^{\beta_{i}} L_{S}^{\alpha(S)}(x)\right),
\end{aligned}
$$

where $S$ ranges over all non-empty subset of $I=\{1,2, \ldots, r\}$ in the summation and

$$
\alpha(S)=m+|S|+\sum_{j \in S} \beta_{j}
$$

Here we describe the analytic continuation of $Z(L, \beta ; s)$. For $\operatorname{Re} s>$ $r+|\beta|$, we have

$$
\begin{aligned}
Z(L, \beta ; s) & \Gamma(s) \\
= & \sum_{n_{1}=1}^{\infty} \cdots \sum_{n_{r}=1}^{\infty} n_{1}^{\beta_{1}} \cdots n_{r}^{\beta_{r}} \int_{0}^{\infty} t^{s-1} e^{-\left(a_{1} n_{1}+\cdots+a_{r} n_{r}+\delta\right) t} d t \\
& =\int_{0}^{\infty} e^{-\delta t} \prod_{j=1}^{r}\left(\sum_{n=1}^{\infty} n^{\beta_{j}} e^{-a_{j} n t}\right) d t .
\end{aligned}
$$

Set

$$
F_{j}(t)=\sum_{n=1}^{\infty} n^{\beta_{j}} e^{-a_{j} n t} \quad \text { and } \quad F(t)=e^{-\delta t} \prod_{j=1}^{r} F_{j}(t)
$$


A term by term differentiation of the identity

$$
\sum_{n=1}^{\infty} e^{-a_{j} n t}=\frac{1}{e^{a_{j} t}-1}, \quad t>0
$$

we get

$$
F_{j}(t)=\left(-a_{j}\right)^{-\beta_{j}}\left(\frac{d}{d t}\right)^{\beta_{j}}\left(\frac{1}{e^{a_{j} t}-1}\right) .
$$

Thus around $t=0, F_{j}(t)$ has the asymptotic expansion

$$
\frac{\beta_{j} !}{\left(a_{j} t\right)^{\beta_{j}+1}}+(-1)^{\beta_{j}} \sum_{n_{j} \geq \beta_{j}+1} \frac{B_{n}\left(a_{j} t\right)^{n-\beta_{j}-1}}{n\left(n-\beta_{j}-1\right) !} .
$$

It follows that at $t=0, F(t)$ has an asymptotic expansion of the form

$$
\sum_{n \geq-(|\beta|+r)} C_{n} t^{n}
$$

Consequently, the analytic continuation of $Z(L, \beta ; s)$ and its special values at negative integers follow from Lemma 7 in Section 4.

When $\beta=0$, we have the following

Corollary. For any integer $m \geq r$, one has

$$
\begin{aligned}
& Z(L, 0 ; r-m) \\
& =\sum_{|\alpha|=m} \frac{(-1)^{m-r-\alpha_{r+1}}(m-r) !}{\alpha_{1} ! \cdots \alpha_{r} ! \alpha_{r+1} !} B_{\alpha_{1}} \cdots B_{\alpha_{r}} a_{1}^{\alpha_{1}-1} \cdots a_{r}^{\alpha_{r}-1} \delta^{\alpha_{r+1}}
\end{aligned}
$$

\subsection{Shintani zeta functions.}

Next we consider another kind of zeta function which were investigated first by Shintani in [8] and then Eie in [3]. Here we reformulate the main result in [3].

Let $A=\left(a_{1}, \ldots, a_{r}\right)$ and $u=\left(u_{1}, \ldots, u_{r}\right)$ be $r$-tuples of complex numbers such that $\operatorname{Re} a_{j}>0$ and $u_{j}>0$. Define the zeta function

$$
Z(A, u ; s)=\sum_{n_{1}=0}^{\infty} \cdots \sum_{n_{r}=0}^{\infty}\left(a_{1}\left(n_{1}+u_{1}\right)+\cdots+a_{r}\left(n_{r}+u_{r}\right)\right)^{-s}
$$


where $\operatorname{Re} s>r$.

Proposition 2. For any integer $m \geq r$, one has

$$
\begin{aligned}
& Z(A, u ; r-m) \\
& \quad=(-1)^{r} \sum_{|p|=m} \frac{(m-r) !}{p_{1} ! \cdots p_{r} !} B_{p_{1}}\left(u_{1}\right) \cdots B_{p_{r}}\left(u_{r}\right) a_{1}^{p_{1}-1} \cdots a_{r}^{p_{r}-1} .
\end{aligned}
$$

Here the summation is over all $p$-tuples of non-negative integers such that and $|p|=p_{1}+\cdots+p_{r}=m$.

\section{Euler's Identity.}

If we start from the fraction

$$
F(T)=\frac{1}{(1-T)^{2}}=\sum_{k=0}^{\infty}(k+1) T^{k},
$$

we obtain the identity

$$
\zeta(s-1)+\zeta(s)=\sum_{n_{1}=1}^{\infty} \sum_{n_{2}=1}^{\infty}\left(n_{1}+n_{2}\right)^{-s}+2 \zeta(s),
$$

from the Dirichlet series $Z_{F}(s)$. Setting $s=2-2 n$, we get Euler's identity

$$
\sum_{k=1}^{n-1} \frac{(2 n) !}{(2 k) !(2 n-2 k) !} B_{2 k} B_{2 n-2 k}=-(2 n+1) B_{2 n}, \quad n \geq 2 .
$$

In this section we shall establish a new identity analogous to that of Euler and then as an illustration of our method we give an extension of the Euler identity to Bernoulli polynomials. We state a lemma.

Lemma 3. Given

$$
P(T)=\sum_{j=0}^{m} b_{j} T^{j}
$$

and

$$
F(T)=\frac{P(T)}{\left(1-T^{m_{1}}\right) \cdots\left(1-T^{m_{r}}\right)}
$$


with $m_{1}+\cdots+m_{r}>m$, then, for $|T|<1$ we have

$$
F(T)=\sum_{j=0}^{m} b_{j} \sum_{n_{1}=0}^{\infty} \cdots \sum_{n_{r}=0}^{\infty} T^{n_{1} m_{1}+\cdots+n_{r} m_{r}+j}
$$

and hence

$$
\begin{aligned}
Z_{F}(s)= & b_{0} \sum_{\substack{n_{1}, \ldots, n_{r} \geq 0 \\
|n|>0}}\left(n_{1} m_{1}+\cdots+n_{r} m_{r}\right)^{-s} \\
& +\sum_{j=1}^{m} b_{j} \sum_{n_{1}, \ldots, n_{r} \geq 0}\left(n_{1} m_{1}+\cdots+n_{r} m_{r}+j\right)^{-s} .
\end{aligned}
$$

To illustrate our principle we consider as a first example, a fraction related to the generating function of the dimensions of Siegel modular forms of genus two,

$$
F(T)=\frac{1}{\left(1-T^{2}\right)\left(1-T^{3}\right)\left(1-T^{5}\right)\left(1-T^{6}\right)}
$$

and we derive a new Bernoulli identity.

Proposition 4. For any integer $m \geq 3$,

$$
\begin{aligned}
& \sum_{|p|=2 m} \frac{(2 m-4) !}{p_{1} ! p_{2} ! p_{3} ! p_{4} !} B_{p_{1}} B_{p_{2}} B_{p_{3}} B_{p_{4}} 2^{p_{1}-1} 3^{p_{2}-1} 5^{p_{3}-1} 6^{p_{4}-1} \\
&=-\frac{1}{1080} \frac{B_{2 m}}{2 m}-\left(\frac{17}{432}+\frac{1}{48} 2^{2 m-2}+\frac{257}{360} 3^{2 m-4}\right) \frac{B_{2 m-2}}{2 m-2} \\
&-\frac{197}{180} \frac{6^{2 m-4}}{2 m-2}\left(B_{2 m-2}\left(\frac{1}{6}\right)+B_{2 m-2}\left(\frac{1}{3}\right)\right) \\
&+\frac{1}{54} \frac{6^{2 m-4}}{2 m-3}\left(25 B_{2 m-3}\left(\frac{1}{6}\right)-16 B_{2 m-3}\left(\frac{1}{3}\right)\right) \\
&-\frac{5^{2 m-3}}{2 m-3} B_{2 m-3}\left(\frac{1}{5}\right) .
\end{aligned}
$$

Proof. Let

$$
F(T)=\frac{1}{\left(1-T^{2}\right)\left(1-T^{3}\right)\left(1-T^{6}\right)\left(1-T^{5}\right)} .
$$


178 M. EIE AND K. F. LAI

By Lemma 3, we have for $\operatorname{Re} s>4$

$$
\begin{aligned}
Z_{F}(s)= & \sum_{\substack{n_{1}, \ldots, n_{4} \geq 0 \\
|n|>0}}\left(2 n_{1}+3 n_{2}+6 n_{3}+5 n_{4}\right)^{-s} \\
= & \sum_{n_{1}=1}^{\infty} \sum_{n_{2}=1}^{\infty} \sum_{n_{3}=1}^{\infty} \sum_{n_{4}=1}^{\infty}\left(2 n_{1}+3 n_{2}+6 n_{3}+5 n_{4}\right)^{-s} \\
& +\sum_{n_{1}=1}^{\infty} \sum_{n_{2}=1}^{\infty} \sum_{n_{3}=1}^{\infty}\left(\left(2 n_{1}+3 n_{2}+6 n_{3}\right)^{-s}+\left(2 n_{1}+6 n_{2}+5 n_{3}\right)^{-s}\right. \\
& \left.+\sum_{n_{1}=1}^{\infty} \sum_{n_{2}=1}^{\infty}\left(\left(2 n_{1}+3 n_{2}\right)^{-s}+\left(2 n_{1}+6 n_{2}\right)^{-s}+\left(2 n_{1}+5 n_{2}\right)^{-s}+5 n_{3}\right)^{-s}+\left(3 n_{1}+6 n_{2}+5 n_{3}\right)^{-s}\right) \\
& +\left(2^{-s}+3^{-s}+6 n^{-s}+5^{-s}\right) \zeta(s) .
\end{aligned}
$$

On the other hand, we decompose $F(T)$ into partial fractions

$$
\begin{aligned}
F(T)= & \frac{1}{180(1-T)^{4}}+\frac{1}{30(1-T)^{3}}+\frac{1}{48(1+T)^{2}}+\frac{1-T^{4}}{5\left(1-T^{5}\right)} \\
& +\frac{19-332 T-23 T^{2}-54 T^{3}+15 T^{4}+144 T^{5}}{720\left(1-T^{6}\right)} \\
& +\frac{514+274 T+514 T^{2}+514 T^{3}+274 T^{4}+514 T^{5}}{720\left(1-T^{6}\right)^{2}} \\
= & \frac{1}{1080} \sum_{k=0}^{\infty}(k+1)(k+2)(k+3) T^{k}+\frac{1}{60} \sum_{k=0}^{\infty}(k+1)(k+2) T^{k} \\
& +\frac{1}{48} \sum_{k=0}^{\infty}(-1)^{k}(k+1) T^{k}+\frac{1}{5} \sum_{k=0}^{\infty}\left(1-T^{4}\right) T^{5 k} \\
& +\frac{1}{720} \sum_{k=0}^{\infty}\left(19-332 T-23 T^{2}-54 T^{3}+15 T^{4}+144 T^{5}\right) T^{6 k} \\
& +\frac{1}{720} \sum_{k=0}^{\infty}(k+1)\left(514+274 T+514 T^{2}\right.
\end{aligned}
$$




$$
\left.+514 T^{3}+274 T^{4}+514 T^{5}\right) T^{6 k}
$$

So the corresponding zeta function is

$$
\begin{aligned}
Z_{F}(s)= & \frac{1}{1080}(\zeta(s-3)+6 \zeta(s-2)+11 \zeta(s-1)+6 \zeta(s)) \\
& +\frac{1}{60}(\zeta(s-2)+3 \zeta(s-1)+2 \zeta(s)) \\
& +\frac{1}{48}\left(\left(2^{2-s}-1\right) \zeta(s-1)+\left(2^{1-s}-1\right) \zeta(s)\right) \\
& +\frac{1}{5}\left(\sum_{k=1}^{\infty}(5 k)^{-s}-\sum_{k=0}^{\infty}(5 k+4)^{-s}\right) \\
& +\frac{1}{720}\left(19 \sum_{k=1}^{\infty}(6 k)^{-s}\right. \\
& -\sum_{k=0}^{\infty}\left(332(6 k+1)^{-s}+23(6 k+2)^{-s}\right. \\
& +\frac{1}{720}\left(514 \sum_{k=1}^{\infty}(6 k)^{-s}\right. \\
& +\sum_{k=0}^{\infty}(k+1)\left(274(6 k+1)^{-s}+514(6 k+2)^{-s}\right. \\
& +514(6 k+3)^{-s}+274(6 k+4)^{-s} \\
& \left.\left.+514(6 k+5)^{-s}\right)\right) .
\end{aligned}
$$

Set $s=4-2 m$ with $m \geq 3$, we get that $Z_{F}(4-2 m)$ is equal to the right hand side of our identity after an elementary calculation.

Consider $Z_{F}(s)$ as a sum of zeta functions associated with linear forms, we have

$$
\begin{aligned}
Z_{F}(4 & -2 m) \\
& =\sum_{|p|=2 m} \frac{(2 m-4) !}{p_{1} ! p_{2} ! p_{3} ! p_{4} !} B_{p_{1}} B_{p_{2}} B_{p_{3}} B_{p_{4}} 2^{p_{1}-1} 3^{p_{2}-1} 6^{p_{3}-1} 5^{p_{4}-1}
\end{aligned}
$$




$$
\begin{aligned}
+\sum_{|p|=2 m-1} \frac{(2 m-4) !}{p_{1} ! p_{2} ! p_{3} !} B_{p_{1}} B_{p_{2}} B_{p_{3}} \\
\cdot\left(2^{p_{1}-1} 3^{p_{2}-1} 5^{p_{3}-1}+2^{p_{1}-1} 3^{p_{2}-1} 6^{p_{3}-1}\right. \\
\left.\quad+2^{p_{1}-1} 6^{p_{2}-1} 5^{p_{3}-1}+3^{p_{1}-1} 6^{p_{2}-1} 5^{p_{3}-1}\right) \\
+\sum_{|p|=2 m-2} \frac{(2 m-4) !}{p_{1} ! p_{2} !} B_{p_{1}} B_{p_{2}} \\
\cdot\left(2^{p_{1}-1} 3^{p_{2}-1}+2^{p_{1}-1} 6^{p_{2}-1}+2^{p_{1}-1} 5^{p_{2}-1}\right. \\
\left.\quad+3^{p_{1}-1} 6^{p_{2}-1}+3^{p_{1}-1} 5^{p_{2}-1}+6^{p_{1}-1} 5^{p_{2}-1}\right)
\end{aligned}
$$

In the second summation, $p=\left(p_{1}, p_{2}, p_{3}\right)$ ranges over all non-negative integers $p_{1}, p_{2}, p_{3}$ such that $p_{1}+p_{2}+p_{3}=2 m-1$. So at least one of $p_{j}$ must be odd. But Bernoulli numbers of odd index are zero except $B_{1}=-1 / 2$. Hence we have

$$
\begin{aligned}
\sum_{|p|=2 m-1} \frac{(2 m-4) !}{p_{1} ! p_{2} ! p_{3} !} B_{p_{1}} B_{p_{2}} B_{p_{3}} a_{1}^{p_{1}-1} a_{2}^{p_{2}-1} a_{3}^{p_{3}-1} \\
=-\frac{1}{2} \sum_{|p|=2 m-2} \frac{(2 m-4) !}{p_{1} ! p_{2} !} B_{p_{1}} B_{p_{2}} \\
\cdot\left(a_{1}^{p_{1}-1} a_{2}^{p_{2}-1}+a_{1}^{p_{1}-1} a_{3}^{p_{2}-1}+a_{2}^{p_{1}-1} a_{3}^{p_{2}-1}\right) .
\end{aligned}
$$

Therefore, the second sum in the summation cancels the third sum. Hence our identity follows.

Remark. Different decompositions of $F(T)$ into partial fractions may lead to different expressions of $Z_{F}(s)$ in terms of finite sums of Riemann zeta functions and Hurwitz zeta functions. However, one can prove that the resulting identities are the same by employing well known identities such as

$$
B_{m}(k \delta)=k^{m-1} \sum_{j=1}^{k} B_{m}\left(\delta+\frac{j}{k}\right) .
$$

The formula in the next proposition is an analogue of Euler's identity. 
Proposition 5. For each positive integer $n \geq 4$, one has

$$
\begin{aligned}
\sum_{k=2}^{n-2} \frac{(2 n-2) !}{(2 k-2) !(2 n-2 k-2) !} \frac{B_{2 k}}{2 k} & \frac{B_{2 n-2 k}}{2 n-2 k} \\
& =\left(-\frac{B_{2 n}}{2 n}\right) \frac{(2 n+1)(2 n-6)}{6(2 n-2)(2 n-3)} .
\end{aligned}
$$

Proof. Let

$$
F(T)=\frac{T^{2}}{(1-T)^{4}}
$$

Then for $|T|<1$,

$$
F(T)=\frac{1}{3 !} \sum_{k=0}^{\infty}(k+1)(k+2)(k+3) T^{k+2}=\frac{1}{6} \sum_{m=0}^{\infty}\left(m^{3}-m\right) T^{m}
$$

The corresponding zeta function $Z_{F}(s)$ is then

$$
\frac{1}{6}(\zeta(s-3)-\zeta(s-1)) .
$$

Also we can express $Z_{F}(s)$ as a sum of zeta functions associated with linear forms. By Lemma 3 we have

$$
Z_{F}(s)=\sum_{n_{1}=0}^{\infty} \sum_{n_{2}=0}^{\infty} \sum_{n_{3}=0}^{\infty} \sum_{n_{4}=0}^{\infty}\left(n_{1}+n_{2}+n_{3}+n_{4}+2\right)^{-s} .
$$

After a change of variables $n_{1}+n_{2}+1=p_{1}, n_{3}+n_{4}+1=p_{2}$ in the summation we get

$$
Z_{F}(s)=\sum_{p_{1}=1}^{\infty} \sum_{p_{2}=1}^{\infty} p_{1} p_{2}\left(p_{1}+p_{2}\right)^{-s}
$$

Set $s=4-2 n$ with $n \geq 4$. The identity

$$
\begin{aligned}
\sum_{k=0}^{2 n-4} \frac{(2 n-4) !}{k !(2 n-k-4) !} \frac{B_{k+2}}{k+2} \frac{B_{2 n-k-2}}{2 n-k-2} & +\left(-\frac{B_{2 n}}{2 n}\right) \frac{2}{(2 n-2)(2 n-3)} \\
& =\frac{1}{6}\left(-\frac{B_{2 n}}{2 n}+\frac{B_{2 n-2}}{2 n-2}\right)
\end{aligned}
$$


follows from Proposition 1 and a simple calculation yields our assertion.

REMARK. The identity of Proposition 5 appears in [7] as a consequence of an identity among Eisenstein series. Similar identities follow from different consideration of generating functions. For example, if we consider $F(T)=T^{3} /(1-T)^{6}$, we get the following identity for $n \geq 6$,

$$
\begin{aligned}
\sum_{\substack{p+q+r=n \\
p, q, r \geq 2}} & \frac{(2 n-6) !}{(2 p-2) !(2 q-2) !(2 r-2) !} \frac{B_{2 p} B_{2 q} B_{2 r}}{8 p q r} \\
= & \left(-\frac{B_{2 n}}{2 n}\right)\left(\frac{1}{120}-\frac{2 n^{2}-5 n}{(2 n-2)(2 n-3)(2 n-4)(2 n-5)}\right) \\
& +\frac{1}{80}\left(\frac{B_{2 n-4}}{2 n-4}\right) .
\end{aligned}
$$

Proposition 6. For any integer $n \geq 2$

$$
\sum_{k=0}^{2 n} \frac{(2 n) !}{k !(2 n-k) !}(-1)^{k} B_{k}(u) B_{2 n-k}(u)=-(2 n-1) B_{2 n} .
$$

Proof. Writing the fraction $F(T)=T /(1-T)^{2}$ in two ways we get the identity

$$
\sum_{k=0}^{\infty} k T^{k}=\sum_{n_{1}=0}^{\infty} \sum_{n_{2}=0}^{\infty} T^{n_{1}+n_{2}+1}
$$

Hence for $\operatorname{Re} s>2$, we have

$$
\begin{aligned}
\zeta(s-1) & =\sum_{n_{1}=0}^{\infty} \sum_{n_{2}=0}^{\infty}\left(n_{1}+n_{2}+1\right)^{-s} \\
& =\sum_{n_{1}=0}^{\infty} \sum_{n_{2}=0}^{\infty}\left(\left(n_{1}+\delta\right)+\left(n_{2}+1-\delta\right)\right)^{-s}, \quad 0<\delta<1 .
\end{aligned}
$$

This is just the function $Z((1,1),(\delta, 1-\delta) ; s)$ of Proposition 2 .

Set $s=2-2 n$, we get

$$
\sum_{k=0}^{2 n} \frac{(2 n) !}{k !(2 n-k) !} B_{k}(\delta) B_{2 n-k}(1-\delta)=-(2 n-1) B_{2 n}
$$


In light of the identity (cf. [14, p. 31])

$$
B_{2 n-k}(1-\delta)=(-1)^{k} B_{2 n-k}(\delta),
$$

we have proved that the identity holds for $0<u<1$.

However, as functions of variable $u$, both sides of the identity are analytic functions of $u$. If it holds for $0<u<1$, it must hold for all $u$.

REMARK. In exactly the same way, we get the following identity

$$
\begin{aligned}
& \quad \sum_{p+q+r=2 n} \frac{(2 n) !}{p ! q ! r !} B_{p}(u) B_{q}(v) B_{r}(w) \\
& =(2 n-1)(2 n-2) B_{2 n}(u+v+w) \\
& \quad+(3-2(u+v+w)) 2 n(2 n-2) B_{2 n-1}(u+v+w) \\
& \quad+\left((u+v+w)^{2}-3(u+v+w)+2\right) 2 n(2 n-1) B_{2 n-2}(u+v+w) .
\end{aligned}
$$

\section{Identities in Ramanujan's notebooks.}

In Chapter 14 of Ramanujan's notebooks II [1], there are many interesting identities on Bernoulli numbers. We shall use here Cauchy's formula for Taylor series coefficients. First we prove a new identity analogous to those of Ramanujan and then we make some remarks on the proof of Ramanujan's identities by our method.

We quote the following classical result from [9]:

Lemma 7. Let $\{\lambda\}$ be a sequence of positive real numbers tending $+\infty$. Suppose that the Dirichlet series

$$
\psi(s)=\sum_{\lambda>0} a_{\lambda} \lambda^{-s},
$$

converges for sufficiently large Re $s$. Let

$$
f(t)=\sum_{\lambda>0} a_{\lambda}^{-\lambda t}
$$

be the corresponding exponential series. If at $t=0, f(t)$ has an expansion of the form

$$
\sum_{n \geq n_{0}} C_{n} t^{n}, \quad n_{0} \text { being integer }
$$


then

1) $\psi(s)$ has a meromorphic continuation in the whole complex plane, and

2) $\psi(-m)=(-1)^{m} m ! C_{m}$ for each integer $m \geq 0$.

Proposition 8. For $\alpha, \beta>0$ with $\alpha \beta=\pi^{2}$ and each positive integer $n$,

$$
\begin{gathered}
\sum_{k=0}^{n+1} \frac{B_{2 k}}{(2 k) !} \frac{B_{2 n+2-2 k}}{(2 n+2-2 k) !}(2 k-1)(2 n-2 k+1)(-\beta)^{k} \alpha^{n+1-k} \\
=(2 n-1) 2^{-1-2 n} \alpha^{1-n} \sum_{k=1}^{\infty} \frac{\operatorname{csch}^{2} k \alpha}{k^{2 n}} \\
+2^{-2 n} \alpha^{2-n} \sum_{k=1}^{\infty} \frac{\operatorname{csch}^{2} k \alpha \operatorname{cotanh} k \alpha}{k^{2 n-1}} \\
+(2 n-1) 2^{-1-2 n}(-\beta)^{1-n} \sum_{k=1}^{\infty} \frac{\operatorname{csch}^{2} k \beta}{k^{2 n}} \\
-2^{-2 n} \beta^{2-n} \sum_{k=1}^{\infty} \frac{\operatorname{csch}^{2} k \beta \operatorname{cotanh} k \beta}{k^{2 n-1}}
\end{gathered}
$$

Proof. For any positive number $\varepsilon$, consider the zeta function

$$
Z_{\varepsilon}(s)=\sum_{n_{1}=1}^{\infty} \sum_{n_{2}=1}^{\infty} n_{1} n_{2}\left(\sqrt{\alpha} n_{1}+(\varepsilon+\sqrt{\beta} i) n_{2}\right)^{-s}, \quad \operatorname{Re} s>4
$$

By Proposition $1, Z_{\varepsilon}(s)$ has an analytic continuation and

$$
\begin{aligned}
Z_{\varepsilon}(2- & 2 n) \\
= & \sum_{k=0}^{2 n-2} \frac{(2 n-2) !}{k !(2 n-2-k) !} \frac{B_{k+2}}{k+2} \frac{B_{2 n-k}}{2 n-k}(\sqrt{\alpha})^{k}(\varepsilon+\sqrt{\beta} i)^{2 n-2-k} \\
& +\left(\frac{(\varepsilon+\sqrt{\beta} i)^{2 n}}{\alpha^{2}}+\frac{\alpha^{n}}{(\varepsilon+\sqrt{\beta} i)^{2}}\right)\left(-\frac{B_{2 n+2}}{2 n+2}\right) \frac{1}{2 n(2 n-1)} .
\end{aligned}
$$


It follows that

$$
\begin{aligned}
I= & \lim _{\varepsilon \rightarrow 0} Z_{\varepsilon}(2-2 n) \\
= & \sum_{k=0}^{n+1} \frac{(2 n-2) !}{(2 k) !(2 n+2-2 k) !}(2 k-1)(2 n-2 k+1) \\
& \cdot B_{2 k} B_{2 n+2-2 k}(-\beta)^{k-1} \alpha^{n-k} .
\end{aligned}
$$

On the other hand, let

$$
\begin{aligned}
F_{\varepsilon}(t) & =\left(\sum_{n_{1}=1}^{\infty} n_{1} e^{-\sqrt{\alpha} n_{1} t}\right)\left(\sum_{n_{2}=1}^{\infty} n_{2} e^{-(\varepsilon+\sqrt{\beta} i) n_{2} t}\right) \\
& =\frac{e^{(\sqrt{\alpha}+\varepsilon+i \sqrt{\beta}) t}}{\left(e^{\sqrt{\alpha} t}-1\right)^{2}\left(e^{(\varepsilon+i \sqrt{\beta}) t}-1\right)^{2}}
\end{aligned}
$$

and

$$
F(t)=\lim _{\varepsilon \rightarrow 0} F_{\varepsilon}(t)=\frac{e^{(\sqrt{\alpha}+i \sqrt{\beta}) t}}{\left(e^{\sqrt{\alpha} t}-1\right)^{2}\left(e^{i \sqrt{\beta} t}-1\right)^{2}} .
$$

Note that for $\operatorname{Re} s>4$,

$$
Z_{\varepsilon}(s) \Gamma(s)=\int_{0}^{\infty} t^{s-1} F_{\varepsilon}(t) d t .
$$

It follows from Lemma 7 that

$$
Z_{\varepsilon}(2-2 n)=(2 n-2) ! \frac{1}{2 \pi i} \int_{|z|=\delta} z^{1-2 n} F_{\varepsilon}(z) d z,
$$

where $0<\delta<1$ and the direction on the circle $|z|=\delta$ is counterclockwise. As $\varepsilon \longrightarrow 0$, we get

$$
I=(2 n-2) ! \frac{1}{2 \pi i} \int_{|z|=\delta} z^{1-2 n} F(z) d z .
$$

Let $C_{N}$ be the contour in the complex plane consisting of the rectangle with vertices $(2 N+1)(\sqrt{\alpha}+\sqrt{\beta} i),(2 N+1)(\sqrt{\alpha}-\sqrt{\beta} i),(2 N+$ 1) $(-\sqrt{\alpha}-\sqrt{\beta} i),(2 N+1)(-\sqrt{\alpha}+\sqrt{\beta} i)$ in counterclock direction. Note that $F(z)$ is bounded on the rectangle by a constant independent of $N$. Thus we have

$$
\lim _{N \rightarrow \infty} \int_{C_{N}} z^{1-2 n} F(z) d z=0 .
$$


This implies in particular that the sum of residues of $z^{1-2 n} F(z)$ inside $C_{N}$ approaches zero as $N \longrightarrow \infty$. Thus the residue at zero is equal to the negative of the sum of residues elsewhere. It follows that

$I=-(2 n-2) ! \sum_{k \neq 0}\left\{\right.$ Residues of $z^{1-2 n} F(z)$ at $\left.z=2 k \sqrt{\beta} i, 2 k \sqrt{\alpha}\right\}$.

Our assertion now follows .

REMARK 1 . When $\alpha=\beta=\pi$ and $n$ is odd, we get

$$
\begin{aligned}
& \sum_{k=0}^{n+1} \frac{B_{2 k} B_{2 n+2-2 k}}{(2 k) !(2 n+2-2 k) !}(2 k-1)(2 n+1-2 k)(-1)^{k} \\
= & (2 n-1)(2 \pi)^{-2 n} \sum_{k=1}^{\infty} \frac{\operatorname{csch}^{2} k \pi}{k^{2 n}}+(2 \pi)^{1-2 n} \sum_{k=1}^{\infty} \frac{\operatorname{csch}^{2} k \pi \operatorname{cotanh} k \pi}{k^{2 n-1}} .
\end{aligned}
$$

REMARK 2. If we consider instead the zeta function

$Z_{\varepsilon}(s)=\sum_{n_{1}=0}^{\infty} \sum_{n_{2}=0}^{\infty}\left(\sqrt{\alpha}\left(n_{1}+u\right)+(\varepsilon+i \sqrt{\beta})\left(n_{2}+v\right)\right)^{-s}, \quad \operatorname{Re} s>2$,

with $0<u, v \leq 1$, we find that for all positive integers $n$

$$
\begin{aligned}
2^{2 n} \sum_{k=0}^{n+1} \frac{B_{2 k}(u)}{(2 k) !} & \frac{B_{2 n-2 k+2}(v)}{(2 n-2 k+2) !} \alpha^{n-k+1}(-\beta)^{k} \\
= & -\frac{1}{2} \alpha^{-n} \sum_{k=1}^{\infty} \frac{\cos (2 k \pi v)\left(e^{2 k u \alpha}+e^{2 k(1-u) \alpha}\right)}{k^{2 n+1}\left(e^{2 k \alpha}-1\right)} \\
& +\frac{1}{2}(-\beta)^{-n} \sum_{k=1}^{\infty} \frac{\cos (2 k \pi u)\left(e^{2 k v \beta}+e^{2 k(1-v) \beta}\right)}{k^{2 n+1}\left(e^{2 k \beta}-1\right)} .
\end{aligned}
$$

Setting $u=v=1 / 2$, we obtain the identity

$$
\begin{gathered}
\alpha^{-n} \sum_{k=1}^{\infty}(-1)^{k+1} \frac{\operatorname{csch}(\alpha k)}{k^{2 n+1}}-(-\beta)^{-n} \sum_{k=1}^{\infty}(-1)^{k+1} \frac{\operatorname{csch}(\beta k)}{k^{2 n+1}} \\
=2^{2 n+1} \sum_{k=0}^{n+1}(-1)^{k} \frac{B_{2 k}\left(\frac{1}{2}\right)}{(2 k) !} \frac{B_{2 n+2-2 k}\left(\frac{1}{2}\right)}{(2 n+2-2 k) !} \alpha^{n+1-k} \beta^{k} .
\end{gathered}
$$


As $u, v$ approach 0 , we get the identity

$$
\begin{aligned}
\alpha^{-n}\left(\frac{1}{2} \zeta(2 n+1)\right. & \left.+\sum_{k=1}^{\infty} \frac{k^{-2 n-1}}{e^{2 k \alpha}-1}\right)-(-\beta)^{-n}\left(\frac{1}{2} \zeta(2 n+1)+\sum_{k=1}^{\infty} \frac{k^{-2 n-1}}{e^{2 \beta k}-1}\right) \\
& =-2^{2 n} \sum_{k=0}^{n+1}(-1)^{k} \frac{B_{2 k}}{(2 k) !} \frac{B_{2 n+2-2 k}}{(2 n+2-2 k) !} \alpha^{n+1-k} \beta^{k}
\end{aligned}
$$

with $n$ a positive integer. The right hand side of the identity we obtained is a constant multiple of

$$
\frac{1}{2 \pi i} \int_{|z|=\delta} \frac{e^{-(\sqrt{\alpha} u+i \sqrt{\beta} v) z} d z}{z^{2 n+1}\left(1-e^{-\sqrt{\alpha} z}\right)\left(1-e^{-i \sqrt{\beta} z}\right)} .
$$

It is zero if $n<-1$. This yields the identity ([1, Chapter 14, p. 261])

$\alpha^{n} \sum_{k=1}^{\infty} \frac{k^{2 n-1}}{e^{2 \alpha k}-1}-(-\beta)^{n} \sum_{k=1}^{\infty} \frac{k^{2 n-1}}{e^{2 \beta k}-1}=\left(\alpha^{n}-(-\beta)^{n}\right) \frac{B_{2 n}}{4 n}, \quad n>1$,

if we let $u, v$ approach zero.

REMARK 3. For each rational function $F(T)$ of the form

$$
\frac{P(T)}{\left(1-T^{m_{1}}\right) \cdots\left(1-T^{m_{r}}\right)}
$$

where $\operatorname{deg} P(T)<m_{1}+\cdots+m_{r}$. The possible poles of $F\left(e^{-z}\right)$ lie in the imaginary axis of the complex plane. By a direct verification, we can find a sequence of contours $C_{N}(N=1,2, \ldots)$ such that the following conditions hold:

1) $C_{N}$ is the rectangle with vertices $x_{N}+i y_{N}, x_{N}-i y_{N},-x_{n}+i y_{N}$, $-x_{N}-i y_{N}, x_{N}>0, y_{N}>0$ with direction counterclockwise,

2) $\lim _{N \rightarrow \infty} x_{N}=\lim _{N \rightarrow \infty} y_{N}=+\infty$,

3) $C_{N}$ does not pass through any pole of $F\left(e^{-z}\right)$, and

4) $\max _{z \in C_{N}}\left|F\left(e^{-z}\right)\right|$ is bounded by a constant independent of $N$.

It follows that for any positive integer $n$,

$$
\lim _{N \rightarrow \infty} \frac{1}{2 \pi i} \int_{C_{N}} z^{-(n+1)} F\left(e^{-z}\right) d z=0 .
$$


This also implies that the residue of $z^{-(n+1)} F\left(e^{-z}\right)$ at 0 is equal to the negative of the sum of residues of $z^{-(n+1)} F\left(e^{-z}\right)$ at $z=2 k \pi i / m_{j}$, $k \in \mathbb{Z}, k \neq 0, j=1, \ldots, r$. Note that the former is a constant multiple of $Z_{F}(-n)$ while the latter is an infinite series in general. This produces an identity between Bernoulli numbers and sums of infinite series. Here we give two examples.

I) For positive integers $m$ and $N$ with $N \geq 3$,

$$
\begin{aligned}
& \sum_{\substack{n=1 \\
n \neq 0}}^{\infty} \frac{\operatorname{cotan}\left(\frac{n \pi}{N}\right)}{n^{2 m+1}} \\
& \quad=\frac{(-1)^{m+1}(2 \pi)^{2 m+1}}{N(2 m+1) !} \sum_{j=1}^{N-2}(N-j-1) B_{2 m+1}\left(\frac{j}{N}\right) .
\end{aligned}
$$

II) For positive integer $m$ and even integer $N \geq 3$,

$$
\begin{aligned}
\sum_{\substack{n=1 \\
n \neq N / 2(\bmod N)}}^{\infty} \frac{\tan \left(\frac{n \pi}{N}\right)}{n^{2 m+1}} \\
=\frac{(-1)^{m}(2 \pi)^{2 m+1}}{N(2 m+1) !} \sum_{j=1}^{N}(-1)^{j}(N-j-1) B_{2 m+1}\left(\frac{j}{N}\right) .
\end{aligned}
$$

\section{Generalizations to several variables.}

It is possible to extend our arguments to the cases when $F(T)$ is a particular type of rational functions of several variables.

Suppose that $\alpha_{j}=\left(\alpha_{j 1}, \ldots, \alpha_{j n}\right), j=1, \ldots, r$ are $n$-tuples of non-negative integers with $\left|\alpha_{j}\right|=\alpha_{j 1}+\cdots+\alpha_{j n}>0$ and $P(T)=$ $P\left(T_{1}, \ldots, T_{n}\right)$ is a polynomial in $n$ variables $T_{1}, \ldots, T_{n}$ with $\operatorname{deg} P(T)<$ $\left|\alpha_{1}\right|+\cdots+\left|\alpha_{r}\right|$. We use the notation

$$
T^{\beta}=\prod_{j=1}^{n} T_{j}^{\beta_{j}}, \quad \text { if } \beta=\left(\beta_{1}, \ldots, \beta_{n}\right) .
$$

Consider the rational function $F(T)$ of the form

$$
\begin{aligned}
F(T) & =\frac{P(T)}{\left(1-T^{\alpha_{1}}\right) \cdots\left(1-T^{\alpha_{r}}\right)} \\
& =\frac{P\left(T_{1}, \ldots, T_{n}\right)}{\left(1-T_{1}^{\alpha_{11}} \cdots T_{n}^{\alpha_{1 n}}\right) \cdots\left(1-T_{1}^{\alpha_{r 1}} \cdots T_{n}^{\alpha_{r n}}\right)} .
\end{aligned}
$$


For $\left|T_{1}\right|<1, \ldots,\left|T_{n}\right|<1, F(T)$ has a power series expansion

$$
\sum_{|\beta|=0}^{\infty} a(\beta) T^{\beta}=\sum_{|\beta|=0}^{\infty} a(\beta) T_{1}^{\beta_{1}} \cdots T_{n}^{\beta_{n}}
$$

For sufficiently large $\operatorname{Re} s$, the zeta function associated with $F(T)$ is given by

$$
Z_{F}(s)=\sum_{\beta_{1}=1}^{\infty} \cdots \sum_{\beta_{n}=1}^{\infty} a(\beta)\left(\beta_{1} \beta_{2} \cdots \beta_{n}\right)^{-s}
$$

Another expression for $Z_{F}(s)$ as a sum of zeta functions associated with linear forms was given by Eie in [2]. This leads to an identity in zeta functions. Using the special values at negative integers, we obtain a family of Bernoulli identities. Here we give an example to illustrate the general procedure.

Consider the rational function

$$
F(T)=\frac{1}{\left(1-T_{1} T_{2}\right)\left(1-T_{1} T_{2}^{2}\right)}
$$

For $\left|T_{1}\right|<1$ and $\left|T_{2}\right|<1$, we have

$$
F(T)=\sum_{n_{1}=0}^{\infty} \sum_{n_{2}=0}^{\infty}\left(T_{1} T_{2}\right)^{n_{1}}\left(T_{1} T_{2}^{2}\right)^{n_{2}}=\sum_{n_{1}=0}^{\infty} \sum_{n_{2}=0}^{\infty} T_{1}^{n_{1}+n_{2}} T_{2}^{n_{1}+2 n_{2}}
$$

It follows for $\operatorname{Re} s>1$,

$$
\begin{aligned}
Z_{F}(s) & =\sum_{n_{1}=1}^{\infty} \sum_{n_{2}=1}^{\infty}\left(\left(n_{1}+n_{2}\right)\left(n_{1}+2 n_{2}\right)\right)^{-s}+\sum_{n_{1}=1}^{\infty} n_{1}^{-2 s}+\sum_{n_{2}=1}^{\infty}\left(2 n_{2}^{2}\right)^{-s} \\
& =\sum_{n_{1}=1}^{\infty} \sum_{n_{2}=1}^{\infty}\left(\left(n_{1}+n_{2}\right)\left(n_{1}+2 n_{2}\right)\right)^{-s}+\left(1+2^{-s}\right) \zeta(2 s)
\end{aligned}
$$

On the other hand, as a rational function of $T_{2}$, we have the following 
190 M. EIE AND K. F. LAI

decomposition of $F(T)$ into partial fraction

$$
\begin{aligned}
F(T)= & \frac{1}{\left(1-T_{1}\right)\left(1-T_{1} T_{2}^{2}\right)}+\frac{T_{1} T_{2}}{\left(1-T_{1}\right)\left(1-T_{1} T_{2}^{2}\right)} \\
& -\frac{T_{1}}{\left(1-T_{1}\right)\left(1-T_{1} T_{2}\right)} \\
= & \sum_{n_{1}=0}^{\infty} \sum_{n_{2}=0}^{\infty} T_{1}^{n_{1}+n_{2}} T_{2}^{2 n_{2}}+\sum_{n_{1}=0}^{\infty} \sum_{n_{2}=0}^{\infty} T_{1}^{n_{1}+n_{2}+1} T_{2}^{2 n_{2}+1} \\
& -\sum_{n_{1}=0}^{\infty} \sum_{n_{2}=0}^{\infty} T_{1}^{n_{1}+n_{2}+1} T_{2}^{n_{2}} .
\end{aligned}
$$

Consequently we get another expression for $Z_{F}(s)$ as

$$
\begin{aligned}
Z_{F}(s)= & \sum_{n_{1}=0}^{\infty} \sum_{n_{2}=1}^{\infty}\left(n_{1}+n_{2}\right)^{-s}\left(2 n_{2}\right)^{-s} \\
& +\sum_{n_{1}=0}^{\infty} \sum_{n_{2}=0}^{\infty}\left(n_{1}+n_{2}+1\right)^{-s}\left(2 n_{2}+1\right)^{-s} \\
& -\sum_{n_{1}=0}^{\infty} \sum_{n_{2}=1}^{\infty}\left(n_{1}+n_{2}+1\right)^{-s} n_{2}^{-s} \\
= & \left(2^{-s}-1\right) \sum_{n_{1}=1}^{\infty} \sum_{n_{2}=1}^{\infty}\left(n_{1}+n_{2}\right)^{-s} n_{2}^{-s}+2^{-s} \zeta(2 s) \\
& +2^{-s} \sum_{n_{1}=0}^{\infty} \sum_{n_{2}=0}^{\infty}\left(\left(n_{1}+\frac{1}{2}\right)+\left(n_{2}+\frac{1}{2}\right)\right)^{-s}\left(n_{2}+\frac{1}{2}\right)^{-s} \\
= & \frac{1}{2}\left(2^{-s}-1\right)\left(\zeta^{2}(s)-\zeta(2 s)\right)+2^{-s} \zeta(2 s) \\
& +2^{-s} \sum_{n_{1}=0}^{\infty} \sum_{n_{2}=0}^{\infty}\left(\left(n_{1}+\frac{1}{2}\right)+\left(n_{2}+\frac{1}{2}\right)\right)^{-s}\left(n_{2}+\frac{1}{2}\right)^{-s}
\end{aligned}
$$

Here we use the identity

$$
\zeta(s)^{2}=\sum_{m=1}^{\infty} \sum_{n=1}^{\infty}(m n)^{-s}
$$




$$
\begin{aligned}
& =\sum_{m=1}^{\infty} \sum_{n=m+1}^{\infty}(m n)^{-s}+\sum_{n=1}^{\infty} \sum_{m=n+1}^{\infty}(m n)^{-s}+\zeta(2 s) \\
& =2 \sum_{n_{1}=1}^{\infty} \sum_{n_{2}=1}^{\infty}\left(n_{1}+n_{2}\right)^{-s} n_{2}^{-s}+\zeta(2 s) .
\end{aligned}
$$

Now it remains to evaluate the zeta functions at negative integers. We need the following proposition from Eie [3].

Proposition 9. Let $Q=a x^{2}+b x y+c y^{2}$ with $a, b, c>0$ and $D=b^{2}-4 a c>0$. Suppose that

$$
Z_{Q}(s)=\sum_{n_{1}=1}^{\infty} \sum_{n_{2}=1}^{\infty}\left(a n_{1}^{2}+b n_{1} n_{2}+c n_{2}^{2}\right)^{-s}, \quad \operatorname{Re} s>1 .
$$

Then $Z_{Q}(s)$ has an analytic continuation and its special value at each negative integer $s=-m(m=1,2, \ldots)$ is given by

$$
\begin{aligned}
Z_{Q}(-m)= & \sum_{p+q+r=m} \frac{m !}{p ! q ! r !} a^{p} b^{q} c^{r} \frac{B_{2 p+q+1}}{2 p+q+1} \frac{B_{2 r+q+1}}{2 r+q+1} \\
& +\left(-\frac{B_{2 m+2}}{2 m+2}\right)\left(\int_{0}^{-b /(2 a)}\left(a x^{2}+b x+c\right)^{m} d x\right. \\
& \left.+\int_{0}^{-b /(2 c)}\left(a+b y+c y^{2}\right)^{m} d y\right) .
\end{aligned}
$$

Proposition 10. Suppose that

$$
Z(s)=\sum_{n_{1}=0}^{\infty} \sum_{n_{2}=0}^{\infty}\left(n_{1}+n_{2}+2 \delta\right)^{-s}\left(n_{2}+\delta\right)^{-s}, \quad \delta>0, \operatorname{Re} s>2 .
$$

Then $Z(s)$ has an analytic continuation and its special value at the negative integer $s=-m(m=1,2, \ldots)$ is given by

$$
\begin{aligned}
& \sum_{k=1}^{m+1} \frac{m !}{(m+1-k) ! k !(2 m+2-k)} B_{k}(\delta) B_{2 m+2-k}(\delta) \\
& \quad+\left(\frac{(-1)^{m}(m !)^{2}}{2(2 m+2) !}+\frac{1}{2(m+1)^{2}}\right) B_{2 m+2}(\delta) .
\end{aligned}
$$


Proof. For Re $s>2$, we have

$$
\begin{aligned}
Z(s)( & \Gamma(s))^{2} \\
& =\sum_{n_{1}=0}^{\infty} \sum_{n_{2}=0}^{\infty} \int_{0}^{\infty} \int_{0}^{\infty}\left(t_{1} t_{2}\right)^{s-1} e^{-\left(n_{1}+n_{2}+2 \delta\right) t_{1}} e^{-\left(n_{2}+\delta\right) t_{2}} d t_{1} d t_{2} \\
& =\int_{0}^{\infty} \int_{0}^{\infty}\left(t_{1} t_{2}\right)^{s-1} \sum_{n_{1}=0}^{\infty} \sum_{n_{2}=0}^{\infty} e^{-\left(n_{1}+n_{2}+2 \delta\right) t_{1}} e^{-\left(n_{2}+\delta\right) t_{2}} d t_{1} d t_{2} \\
& =\int_{0}^{\infty} \int_{0}^{\infty}\left(t_{1} t_{2}\right)^{s-1} \frac{e^{(1-\delta) t_{1}+t_{2}} e^{(1-\delta) t_{1}}}{\left(e^{t_{1}+t_{2}}-1\right)\left(e^{t_{1}}-1\right)} d t_{1} d t_{2} \\
& =\int_{0}^{\infty} \frac{t^{2 s-1} e^{(1-\delta) t}}{e^{t}-1} d t \int_{0}^{1} \frac{(u(1-u))^{s-1} e^{(1-\delta) t u}}{e^{t u}-1} d u
\end{aligned}
$$

Rewrite the above formula as

$$
Z(s) \Gamma(s)=\int_{0}^{\infty} t^{2 s-3} \frac{t e^{(1-\delta) t}}{e^{t}-1} d t \frac{1}{\Gamma(s)} \int_{0}^{1}(u(1-u))^{s-1} \frac{e^{(1-\delta) t u}}{e^{t u}-1} d u .
$$

It follows from a standard process as given in the proof of the main theorem in [3] that

$$
Z(-m)=\frac{(-1)^{m} m !}{2} \sum_{\beta=0}^{2 m+2} \frac{B_{2 m+2-\beta}(1-\delta) B_{\beta}(1-\delta)}{(2 m+2-\beta) ! \beta !} F_{\beta}(-m),
$$

where

$$
F_{\beta}(s)=\frac{1}{\Gamma(s)} \int_{0}^{1}(u(1-u))^{s-1} u^{\beta-1} d u=\frac{\Gamma(s+\beta-1)}{\Gamma(2 s+\beta-1)} .
$$

An elementary calculation shows that

$$
F_{\beta}(-m)= \begin{cases}m !, & \text { if } \beta=2 m+2, \\ 2(-1)^{m} \frac{(2 m+1-\beta) !}{(m+1-\beta) !}, & \text { if } 0 \leq \beta \leq m+1, \\ 0, & \text { if } m+2 \leq \beta \leq 2 m+1 .\end{cases}
$$

Hence our assertion follows. 
Using the identity

$$
\begin{aligned}
\sum_{n_{1}=1}^{\infty} \sum_{n_{2}=1}^{\infty}\left(\left(n_{1}\right.\right. & \left.\left.+n_{2}\right)\left(n_{1}+2 n_{2}\right)\right)^{-s}+\left(1+2^{-s}\right) \zeta(2 s) \\
= & \frac{1}{2}\left(2^{-s}-1\right)\left(\zeta^{2}(s)-\zeta(2 s)\right)+2^{-s} \zeta(2 s) \\
& +2^{-s} \sum_{n_{1}=0}^{\infty} \sum_{n_{2}=0}^{\infty}\left(\left(n_{1}+\frac{1}{2}\right)+\left(n_{2}+\frac{1}{2}\right)\right)^{-s}\left(n_{2}+\frac{1}{2}\right)^{-s}
\end{aligned}
$$

and propositions 9 and 10, we get the following Bernoulli identity

$$
\begin{aligned}
& \sum_{p+q+r=m} \frac{m ! 3^{q} 2^{r}}{p ! q ! r !} \frac{B_{2 p+q+1}}{2 p+q+1} \frac{B_{2 r+q+1}}{2 r+q+1} \\
&+\left(\frac{-B_{2 m+2}}{2 m+2}\right)\left(\int_{0}^{-3 / 2}\left(x^{2}+3 x+2\right)^{m} d x\right. \\
&\left.\quad+\int_{0}^{-3 / 4}\left(1+3 y+2 y^{2}\right)^{m} d y\right) \\
&= \frac{1}{2}\left(2^{m}-1\right)\left(\frac{B_{m+1}}{m+1}\right)^{2} \\
&+2^{m-1}\left(\frac{(-1)^{m}(m !)^{2}}{(2 m+2) !}+\frac{1}{(m+1)^{2}}\right) B_{2 m+2}\left(\frac{1}{2}\right) \\
&+2^{m} \sum_{k=1}^{[(m+1) / 2]} \frac{m !}{(m+1-2 k) !(2 k) !(2 m+2-2 k)} \\
& \cdot B_{2 k}\left(\frac{1}{2}\right) B_{2 m+2-2 k}\left(\frac{1}{2}\right) \cdot
\end{aligned}
$$

REMARK 1. As shown above, the consideration of cases of several variables leads to zeta functions with products of linear forms. Though we have no general formula to evaluate their special values at negative integers, it is possible to calculate these values case by case.

REMARK 2. It is possible to further extend our arguments in this section to the cases that

$$
F(T)=\frac{P(T)}{\left(1-T^{\alpha_{1}}\right) \cdots\left(1-T^{\alpha_{r}}\right)},
$$


with $P(T)$ not necessarily a polynomial and $\alpha_{1}, \ldots, \alpha_{r}$ are not necessarily $n$-tuples of non-negative integers. Indeed, we only need the following considerations.

I) $P(T)$ is a finite complex linear conbination of $T_{1}^{\beta_{1}} \cdots T_{n}^{\beta_{n}}$ with $\operatorname{Re} \beta_{j} \geq 0$.

II) For all $1 \leq j \leq r, \alpha_{j}=\left(\alpha_{j 1}, \ldots, \alpha_{j n}\right)$ with $\alpha_{j i}=0$ or $\operatorname{Re}$ $\alpha_{j i}>0$, but $\alpha_{j} \neq 0$.

Under the second condition, for $0 \leq T_{j}<1$, we have the expansion

$$
\begin{aligned}
P(T) & =\sum_{m_{1}=0}^{\infty} \cdots \sum_{m_{r}=0}^{\infty} P(T) T^{m_{1} \alpha_{1}+\cdots+m_{r} \alpha_{r}} \\
& =\sum_{m_{1}=0}^{\infty} \cdots \sum_{m_{r}=0}^{\infty} P(T) \prod_{j=1}^{n} T_{j}^{m_{1} \alpha_{j 1}+\cdots+m_{r} \alpha_{j r}} .
\end{aligned}
$$

Hence it is easy to write down $Z_{F}(s)$ as a sum of zeta functions associated with products of $n$ linear forms. By employing the same arguments as in Proposition 8 we obtain more identities. As an example we consider the function

$$
F(T)=\frac{T^{(u \sqrt{\alpha}+v(\varepsilon+i \sqrt{\beta}))}}{\left(1-T^{\sqrt{\alpha}}\right)\left(1-T^{\varepsilon+i \sqrt{\beta}}\right)},
$$

where $\varepsilon>0,0<u, v<1$ and $\alpha, \beta>0$ with $\alpha \beta=\pi^{2}$. Calculating the residues and separating the real and imaginary parts we obtain the following known ( $c f .[1$, volume II, p. 276]) identity

$$
\begin{aligned}
2^{2 m} \sum_{k=0}^{m+1} \frac{B_{2 n-2 k+2}(u) B_{2 k}(v)}{(2 n-2 k+2) !(2 k) !} \alpha^{m+1-k}(-\beta)^{k} \\
=-\frac{1}{2} \alpha^{-m} \sum_{k=1}^{\infty} \frac{\cos (2 k \pi v)\left(e^{2 k u \alpha}+e^{2 k(1-u) \alpha}\right)}{k^{2 m+1}\left(e^{2 k \alpha}-1\right)} \\
\quad+\frac{1}{2}(-\beta)^{m} \sum_{k=1}^{\infty} \frac{\cos (2 k \pi u)\left(e^{2 k v \beta}+e^{2 k(1-v) \beta}\right)}{k^{2 m+1}\left(e^{2 k \beta}-1\right)} .
\end{aligned}
$$




\section{Part II}

\section{Minking Eie}

Throughout the rest of the paper, we use the following notations: $p$ is an odd prime number, $m, n$ are integers such that $p-1$ is not a divisor of $m, N$ is a positive integer or zero.

\section{An identity for zeta functions.}

We apply the method of Part I to establish an identity for zeta functions.

Proposition 11. For any prime number $p$ and complex number $s$ with Re $s>1$, one has

$$
\left(1-p^{-s}\right) \zeta(s)=p^{-(N+1) s} \sum_{\substack{(j, p)=1 \\ 1 \leq j<p^{N+1}}} \zeta\left(s ; \frac{j}{p^{N+1}}\right)
$$

Proof. Consider the zeta function $Z_{F}(s)$ associated with the rational function

$$
F(T)=\frac{1}{1-T}-\frac{1}{1-T^{p}}
$$

It is easy to see that for $\operatorname{Re} s>1$

$$
Z_{F}(s)=\sum_{k=1}^{\infty} k^{-s}-\sum_{k=1}^{\infty}(k p)^{-s}=\left(1-p^{-s}\right) \zeta(s)
$$


On the other hand, we have for any nonegative integer $N$,

$$
\begin{aligned}
F(T) & =\frac{T-T^{p}}{(1-T)\left(1-T^{p}\right)} \\
& =\frac{T+T^{2}+\cdots+T^{p-1}}{1-T^{p}} \\
& =\frac{\left(T+T^{2}+\cdots+T^{p-1}\right)\left(1+T^{p}+T^{2 p}+\cdots+T^{p\left(p^{N}-1\right)}\right)}{\left(1-T^{p^{N+1}}\right)} \\
& =\sum_{\substack{(j, p)=1 \\
1 \leq j<p^{N+1}}} \sum_{k=0}^{\infty} T^{j+k p^{N+1}} .
\end{aligned}
$$

It follows that for $\operatorname{Re} s>1$,

$$
Z_{F}(s)=p^{-(N+1) s} \sum_{\substack{(j, p)=1 \\ 1 \leq j<p^{N+1}}} \zeta\left(s ; \frac{j}{p^{N+1}}\right)
$$

Note that $Z_{F}(s)$ is determined by $F(T)$ uniquely through the integral formula

$$
Z_{F}(s) \Gamma(s)=\int_{0}^{\infty} t^{s-1} F\left(e^{-t}\right) d t, \quad \operatorname{Re} s>1,
$$

where $\Gamma(s)$ is the classical gamma function. Thus our identity follows.

As a consequence, we have the following.

Proposition 12. Suppose $m$ is a positive even integer and $p$ is an odd prime with $p-1$ not a divisor of $m$. Then

$$
\left(1-p^{m-1}\right) \frac{B_{m}}{m} \equiv C_{0}(m)+C_{1}(m) \quad\left(\bmod p^{N+1}\right),
$$

where

$$
C_{l}(m)=\frac{1}{m} \sum_{\substack{(j, p)=1 \\
1 \leq j<p^{N+1}}} j^{m-l}\left(\begin{array}{c}
m \\
l
\end{array}\right) B_{l} p^{(N+1)(l-1)}, \quad 0 \leq l \leq m .
$$


Proof. We begin with the identity in Proposition 11. Both the Riemann zeta function $\zeta(s)$ and Hurwitz zeta functions

$$
\zeta\left(s ; \frac{j}{p^{N+1}}\right), \quad j=1, \ldots, p^{N+1},
$$

have analytic continuations in the whole complex plane. So that the identity I) is true for all $s$. In particular, we can set $s=1-m$ in the identity to yield

$$
\begin{aligned}
\left(1-p^{m-1}\right) \frac{B_{m}}{m} & =\frac{1}{m} \sum_{\substack{(j, p)=1 \\
1 \leq j<p^{N+1}}} p^{(N+1)(m-1)} B_{m}\left(\frac{j}{p^{N+1}}\right) \\
& =\frac{1}{m} \sum_{\substack{(j, p)=1 \\
1 \leq j<p^{N+1}}} \sum_{l=0}^{m}\left(\begin{array}{c}
m \\
l
\end{array}\right) B_{l} j^{m-l} p^{(N+1)(l-1)} \\
& =\sum_{l=0}^{m} C_{l}(m) .
\end{aligned}
$$

Note that the exponent of $p$ occurs in $l$ ! is not greater than

$$
\frac{l}{p}+\frac{l}{p^{2}}+\cdots+\frac{l}{p^{l}}+\cdots=\frac{l}{p-1} \leq \frac{l}{2} .
$$

Also $p B_{l}$ is $p$-integral for all $l$ and

$$
C_{l}(m)=(m-1) \cdots(m-l+1) \sum_{\substack{(j, p)=1 \\ 1 \leq j<p^{N+1}}} j^{m-l} \frac{1}{l !}\left(p B_{l}\right) p^{(N+1)(l-1)-1}
$$

Thus $C_{l}(m) \equiv 0\left(\bmod p^{N+1}\right)$ provides that

$$
-\frac{l}{2}+(N+1)(l-1)-1 \geq N+1 .
$$

This is equivalent to

$$
(N+1)(l-2) \geq \frac{l}{2}+1 .
$$


But $N$ is a nonnegative integer, the inequality holds provides that

$$
l-2 \geq \frac{l}{2}+1
$$

This is equivalent to $l \geq 6$. Thus it follows

$$
\begin{aligned}
\left(1-p^{m-1}\right) \frac{B_{m}}{m} & \equiv \sum_{l=0}^{5} C_{l}(m)\left(\bmod p^{N+1}\right) \\
& \equiv C_{0}(m)+C_{1}(m)+C_{2}(m)+C_{4}(m)\left(\bmod p^{N+1}\right) .
\end{aligned}
$$

Next we prove

$$
C_{2}(m) \equiv 0 \quad\left(\bmod p^{N+1}\right)
$$

and

$$
C_{4}(m) \equiv 0 \quad\left(\bmod p^{N+1}\right)
$$

Note that

$$
C_{2}(m)=\frac{m-1}{2} B_{2} \sum_{(j, p)=1} j^{m-2} p^{N+1}=\frac{m-1}{12} p^{N+1} \sum_{(j, p)=1} j^{m-2}
$$

If $p \neq 3$, then

$$
\frac{m-1}{12} p^{N+1}
$$

is $p$-integral and divisible by $p^{N+1}$. However the case $p=3$ is impossible under the assumption that $p-1$ is not a divisor of $m$. This proves that

$$
C_{2}(m) \equiv 0 \quad\left(\bmod p^{N+1}\right) .
$$

Now consider the case $l=4$,

$$
\begin{aligned}
C_{4}(m) & =\frac{(m-1)(m-2)(m-3)}{24} B_{4} \sum_{(j, p)=1} j^{m-4} p^{3 N+3} \\
& =-\frac{(m-1)(m-2)(m-3)}{720} p^{3 N+3} \sum_{(j, p)=1} j^{m-4} \\
& =-\frac{(m-1)(m-2)(m-3)}{2^{4} 3^{2} 5} p^{3 N+3} \sum_{(j, p)=1} j^{m-4} .
\end{aligned}
$$


Obviously $C_{4}(m)$ is $p$-integral and divisible by $p^{N+1}$ for any odd prime $p$. Hence we can drop the last two terms in our congruence relation and it completes our proof.

\section{Congruence relations of $C_{0}(m)$.}

Recall that for $0 \leq l \leq m$,

$$
C_{l}(m)=\frac{1}{m} \sum_{\substack{(j, p)=1 \\
1 \leq j<p^{N+1}}} j^{m-l}\left(\begin{array}{c}
m \\
l
\end{array}\right) B_{l} p^{(N+1)(l-1)} .
$$

As shown in Proposition 12, Kummer's congruences are equivalent to

$$
C_{0}(m)+C_{1}(m) \equiv C_{0}(n)+C_{1}(n) \quad\left(\bmod p^{N+1}\right) .
$$

However

$$
C_{1}(m)=-\frac{1}{2} \sum_{\substack{(j, p)=1 \\ 1 \leq j<p^{N+1}}} j^{m-1}
$$

So it is easy to see that if $m \equiv n\left(\bmod (p-1) p^{N}\right)$, then

$$
C_{1}(m) \equiv C_{1}(n) \quad\left(\bmod p^{N+1}\right),
$$

since

$$
j^{m-1} \equiv j^{n-1} \quad\left(\bmod p^{N+1}\right),
$$

for all integer $j$ relative prime to $p$. Consequently, Kummer's congruences are equivalent to

$$
\frac{p^{-(N+1)}}{m} \sum_{\substack{(j, p)=1 \\ 1 \leq j<p^{N+1}}} j^{m} \equiv \frac{p^{-(N+1)}}{n} \sum_{\substack{(j, p)=1 \\ 1 \leq j<p^{N+1}}} j^{n} \quad\left(\bmod p^{N+1}\right) .
$$

To simplify the notation we write

$$
\sum_{(j, p)=1}
$$


for

$$
\sum_{\substack{(j, p)=1 \\ 1 \leq j<p^{N+1}}}
$$

Our proof that

$$
C_{0}(m) \equiv C_{0}(n) \quad\left(\bmod p^{N+1}\right),
$$

employs the classical theorems of Fermat ([6, Theorems 71,88$])$.

Proposition 13. Suppose that $m, n$ are positive even integers and $p$ is an odd prome with $p-1$ not a divisor of $m$. Then

$$
C_{0}(m) \equiv C_{0}(n) \quad\left(\bmod p^{N+1}\right),
$$

if $m \equiv n\left(\bmod (p-1) p^{N}\right)$.

Proof. By the fundamental theorem of finite abelian group ([11]), we can decompose the multiplicative group $G=\left(\mathbb{Z} / p^{N+1} \mathbb{Z}\right)^{*}$ into a direct product

$$
G_{0} \prod_{i=1}^{\mu} G_{i}
$$

where $G_{0}$ is a cyclic group of order $p-1$ and $G_{i}(i=1, \ldots, \mu)$ is a cyclic group of order $p^{e_{i}}$ with

$$
e_{1}+\cdots+e_{\mu}=N \text {. }
$$

Such a decomposition is possible since $\mathbb{Z} / p^{N+1} \mathbb{Z}$ contains $\mathbb{Z} / p \mathbb{Z}$ as a subfield and the multiplicative group $(\mathbb{Z} / p \mathbb{Z})^{*}$ is a cyclic group of order $p-1([8])$.

Suppose that $g, g_{1}, \ldots, g_{\mu}$ are generators of $G_{0}, G_{1}, \ldots, G_{\mu}$; respectively. It follows

$$
\begin{aligned}
C_{0}(m) & =\frac{p^{-(N+1)}}{m} \sum_{(j, p)=1} j^{m} \\
& =\frac{p^{-(N+1)}}{m}\left(1+g^{m}+\cdots+g^{m(p-2)}\right) \prod_{i=1}^{\mu}\left(1+g_{i}^{m}+\cdots+g_{i}^{m\left(p^{e_{i}}-1\right)}\right) .
\end{aligned}
$$

Note that $g^{m} \neq 1$ since $p-1$ is not a divisor of $m$. So

$$
1+g^{m}+\cdots+g^{m(p-2)}=\frac{g^{(p-1) m}-1}{g^{m}-1} .
$$


For each $1 \leq i \leq \mu$, if $g_{i}^{m}=1$, then we automatically have

$$
1+g_{i}^{m}+\cdots+g_{i}^{m\left(p^{e_{i}}-1\right)}=p^{e_{i}} .
$$

If $g_{i}^{m} \neq 1$, we have

$$
1+g_{i}^{m}+\cdots+g_{i}^{m\left(p^{e_{i}}-1\right)}=\frac{g_{i}^{m p^{e_{i}}}-1}{g_{i}^{m}-1} .
$$

But

$$
g_{i}^{m p^{e_{i}}} \equiv 1 \quad\left(\bmod p^{e_{i}}\right) .
$$

Consequently the sum

$$
1+g_{i}^{m}+\cdots+g_{i}^{m\left(p^{e_{i}}-1\right)}
$$

always has the divisor $p^{e_{i}}$.

With a possible permutation in the indices, we suppose that $g_{i}^{m}=1$ for $1 \leq i<q$ and $g_{i}^{m} \neq 1$ for $q \leq i \leq \mu$. Then we rewrite $C_{0}(m)$ as

$$
C_{0}(m)=\frac{p^{-(N+1)}}{m} \frac{g^{m(p-1)}-1}{g^{m}-1} \prod_{i=1}^{q-1} p^{e_{i}} \prod_{i=q}^{\mu} \frac{g_{i}^{m p^{e_{i}}}-1}{g_{i}^{m}-1}
$$

Suppose that $g^{p-1}=1+k p$, then it is a direct verification that $\left(g^{m(p-1)}-1\right) /(m p)$ is $p$ integral and

$$
\frac{1}{m p}\left(g^{m(p-1)}-1\right) \equiv k \quad(\bmod p) .
$$

It follows

$$
\frac{1}{m p}\left(g^{m(p-1)}-1\right) \equiv \frac{1}{n p}\left(g^{n(p-1)}-1\right) \quad(\bmod p)
$$

Also $g^{m}-1$ and $g_{i}^{m}-1(i=q, \ldots, \mu)$ are invertible elements of $\mathbb{Z} / p^{N+1} \mathbb{Z}$ and

$$
\begin{aligned}
& g^{m}-1 \equiv g^{n}-1\left(\bmod p^{N+1}\right), \\
& g_{i}^{m}-1 \equiv g_{i}^{n}-1\left(\bmod p^{N+1}\right) .
\end{aligned}
$$


So that

$$
\begin{aligned}
& \left(g^{m}-1\right)^{-1} \equiv\left(g^{n}-1\right)^{-1}\left(\bmod p^{N+1}\right), \\
& \left(g_{i}^{m}-1\right)^{-1} \equiv\left(g_{i}^{n}-1\right)^{-1}\left(\bmod p^{N+1}\right) .
\end{aligned}
$$

Multiply all these congruences together, we get

$$
C_{0}(m) \equiv C_{0}(n) \quad\left(\bmod p^{N+1}\right) .
$$

\section{8. von Staudt's Theorem.}

Our proof of Proposition 13 is analogous to the proof of von Staudt's Theorem in [2, p. 384]. Indeed we are able to give another proof of von Staudt's Theorem by the identity (II) with $N=0$. In other words, we are able to kill two birds with one stone.

Proposition 14 (von Staudt's Theorem). Suppose that $m$ is a positive even integer and $p$ is an odd prime. Then

a) $B_{m}$ is p-integral if $p-1$ is not a divisor of $m$,

b) if $p-1$ is a divisor of $m$, then $p B_{m}$ is p-integral and

$$
p B_{m} \equiv-1 \quad(\bmod p)
$$

Proof. We begin with the identity (II) with $N=0$.

$$
\left(1-p^{m-1}\right) \frac{B_{m}}{m}=\frac{1}{m} \sum_{j=1}^{p-1} \sum_{l=0}^{m}\left(\begin{array}{c}
m \\
l
\end{array}\right) B_{l} j^{m-l} p^{l-1}
$$

Multiply both sides by $m$, we get

$$
\begin{aligned}
\left(1-p^{m-1}\right) B_{m} & =\sum_{j=1}^{p-1} \sum_{l=0}^{m}\left(\begin{array}{c}
m \\
l
\end{array}\right) B_{l} j^{m-l} p^{l-1} \\
& =\sum_{j=1}^{p-1} \sum_{l=0}^{m-1}\left(\begin{array}{c}
m \\
l
\end{array}\right) B_{l} j^{m-l} p^{l-1}+p^{m-1}(p-1) B_{m} .
\end{aligned}
$$


It follows

$$
\left(1-p^{m}\right) B_{m}=\sum_{j=1}^{p-1} \sum_{l=0}^{m-1}\left(\begin{array}{c}
m \\
l
\end{array}\right) B_{l} j^{m-l} p^{l-1} .
$$

Now we shall prove our assertion by induction on $m$.

Suppose that $p B_{l}$ is $p$-integral for all $1 \leq l<m-1$. Then

$$
\left(\begin{array}{c}
m \\
l
\end{array}\right) B_{l} p^{l-1}=\left(\begin{array}{c}
m \\
l
\end{array}\right)\left(p B_{l}\right) p^{l-2}
$$

is $p$-integral provide that $l \geq 2$. Hence we have

$$
\left(1-p^{m}\right) B_{m} \equiv \frac{1}{p} \sum_{j=1}^{p-1} j^{m}-\frac{m}{2} \sum_{j=1}^{p-1} j^{m-1}+\frac{m(m-1)}{12} p \sum_{j=1}^{p-1} j^{m-2} \quad(\bmod p) .
$$

Note that $p \neq 2$ or 3 , so that the third term on the right hand side is also $p$-integral and divisible by $p$. So we can drop it in our consideration. follows

If $(p-1)$ is a divisor of $m$, then $j^{m}=1$ for all $1 \leq j \leq p-1$. It

$$
\left(1-p^{m}\right) B_{m} \equiv \frac{p-1}{p}-\frac{m}{2} \sum_{j=1}^{p-1} j^{m-1} \quad(\bmod p) .
$$

Thus $p B_{m}$ is $p$-integral and

$$
p B_{m} \equiv-1 \quad(\bmod p) .
$$

On the other hand, if $p-1$ is not a divisor of $m$, we choose an element $g$ of order $p-1$ in $(\mathbb{Z} / p \mathbb{Z})^{*}$. Then

$$
\begin{aligned}
\left(1-p^{m}\right) B_{m} & \equiv \frac{1}{p} \sum_{j=0}^{p-2} g^{m j}-\frac{m}{2} \sum_{j=1}^{p-1} g^{(m-1) j}(\bmod p) \\
& =\frac{1}{p} \frac{g^{(p-1) m}-1}{g^{m}-1}-\frac{m}{2} \sum_{j=1}^{p-1} g^{(m-1) j}(\bmod p)
\end{aligned}
$$

Suppose that $g^{p-1}=1+\alpha p$. Then

$$
g^{(p-1) m} \equiv 1+m \alpha p \quad\left(\bmod p^{2}\right) .
$$

Thus

$$
\frac{1}{p} \frac{g^{(p-1) m}-1}{g^{m}-1}
$$


is $p$-integral. This proves that $B_{m}$ is $p$-integral.

\section{A slight generalization of Kummer's congruences.}

Here we reformulate Kummer's congruences in a general form.

Theorem 15. Suppose that $m, n$ are positive even integers and $k$ is a positive integer such that $p-1$ is not a divisor of $m$ for all prime divisor $p$ of $k$. Then

$$
\frac{B_{m}}{m} \prod_{p \mid k}\left(1-p^{m-1}\right) \equiv \frac{B_{n}}{n} \prod_{p \mid k}\left(1-p^{n-1}\right) \quad(\bmod k),
$$

if $m \equiv n(\bmod \varphi(k))$, here $\varphi$ is the Euler $\varphi$-function.

Proof. Suppose that

$$
k=\prod_{i=1}^{\mu} p_{i}^{N_{i}+1}
$$

with $p_{1}, \ldots, p_{\mu}$ are distinct prime numbers and $N_{1}, \ldots, N_{\mu}$ are nonnegative integers.

Consider the zeta function

$$
\zeta_{k}(s)=\sum_{\substack{(n, k)=1 \\ n \geq 1}} n^{-s}, \quad \operatorname{Re} s>1 .
$$

$\zeta_{k}(s)$ has the Euler product

$$
\prod_{p \mid k}\left(1-p^{-s}\right) \zeta(s)
$$

As usual, $\zeta_{k}(s)$ has its analytic continuation and its special value at $s=1-m$ is given by

$$
\zeta_{k}(1-m)=-\frac{B_{m}}{m} \prod_{p \mid k}\left(1-p^{m-1}\right) .
$$

On the other hand, $\zeta_{k}(s)$ is the zeta function associated with the rational function

$$
F(T)=\frac{1}{1-T}-\sum_{i=1}^{\mu} \frac{1}{1-T^{p_{i}}}+\sum_{\substack{1 \leq i \\ l \leq \mu}} \frac{1}{1-T^{p_{i} p_{l}}}+\cdots+(-1)^{\mu} \frac{1}{1-T^{p_{1} \cdots p_{\mu}}}
$$


by the well known inclusion-exclusion principle. Also for $|T|<1, F(T)$ has the power series expansion

$$
F(T)=\sum_{\substack{(j, k)=1 \\ 1 \leq j<k}} \sum_{\substack{l=0 \\ 1<k}}^{\infty} T^{j+l k}
$$

Thus it follows

$$
\zeta_{k}(s)=k^{-s} \sum_{\substack{(j, p)=1 \\ 1 \leq j<k}} \zeta\left(s ; \frac{j}{k}\right)
$$

and hence

$$
\zeta_{k}(1-m)=-\frac{k^{m-1}}{m} \sum_{\substack{(j, k)=1 \\
1 \leq j<k}} B_{m}\left(\begin{array}{l}
j \\
k
\end{array}\right)=-\frac{1}{m} \sum_{\substack{(j, k)=1 \\
1 \leq j<k}} \sum_{l=0}^{m}\left(\begin{array}{c}
m \\
l
\end{array}\right) j^{m-l} B_{l} k^{l-1} .
$$

Set

$$
C_{l}(m)=\frac{1}{m} \sum_{\substack{(j, k)=1 \\
1 \leq j<k}}\left(\begin{array}{c}
m \\
l
\end{array}\right) j^{m-l} B_{l} k^{l-1}
$$

Note that for each $1 \leq i \leq \mu$

$$
C_{l}(m) \equiv \frac{1}{m p_{i}^{N_{i}+1}} \sum_{\substack{(j, k)=1 \\
1 \leq j<p^{N_{i}+1}}}\left(\begin{array}{c}
m \\
l
\end{array}\right) j^{m-l} B_{l} k^{l-1} \quad\left(\bmod p_{i}^{N_{i}+1}\right) .
$$

By our proof Proposition 2, we have for $l \geq 2$,

$$
C_{l}(m) \equiv 0 \quad\left(\bmod p_{i}^{N_{i}+1}\right), \quad i=1, \ldots, \mu .
$$

By Chinese remainder's theorem, we get for $l \geq 2$.

$$
C_{l}(m) \equiv 0 \quad(\bmod k)
$$

This implies

$$
\frac{B_{m}}{m} \prod_{p \mid k}\left(1-p^{m-1}\right) \equiv \frac{1}{m k} \sum_{\substack{(j, k)=1 \\ 1 \leq j<k}} j^{m}-\frac{1}{2} \sum_{\substack{(j, k)=1 \\ 1 \leq j<k}} j^{m-1}(\bmod k) .
$$


Consequently our assertion is equivalent to prove

$$
\frac{1}{m k} \sum_{\substack{(j, k)=1 \\ 1 \leq j<k}} j^{m} \equiv \frac{1}{n k} \sum_{\substack{(j, k)=1 \\ 1 \leq j<k}} j^{n} \quad(\bmod k) .
$$

But it follows from the fact that

$$
\frac{1}{m k} \sum_{\substack{(j, k)=1 \\ 1 \leq j<k}} j^{m} \equiv \frac{p_{i}^{-\left(N_{i}+1\right)}}{m} \sum_{\substack{(j, k)=1 \\ 1 \leq j<p^{N_{i}+1}}} j^{m} \quad\left(\bmod p^{N_{i}+1}\right)
$$

and our previous identity

$$
\frac{p_{i}^{-\left(N_{i}+1\right)}}{m} \sum_{\substack{(j, k)=1 \\ 1 \leq j<p^{N_{i}+1}}} j^{m} \equiv \frac{p_{i}^{-\left(N_{i}+1\right)}}{n} \sum_{\substack{(j, k)=1 \\ 1 \leq j<p^{N_{i}+1}}} j^{n}\left(\bmod p^{N_{i}+1}\right),
$$

for all $1 \leq i \leq \mu$.

\section{0. $p$-adic interpolation.}

Let $p$ be a prime number. $\mathbb{Z}_{p}$ and $\mathbb{Q}_{p}$ are the ring of $p$-adic integers and the field of $p$-adic numbers, respectively. $\Omega_{p}$ is the algebra completion of $\mathbb{Q}_{p}$. For a fixed positive integer $k$, we let $X_{k}$ be the inverse projective limit of $\mathbb{Z} / k p^{N} \mathbb{Z}$, i.e.

$$
X_{k}=\lim _{\leftarrow-} \mathbb{Z} / k p^{N} \mathbb{Z},
$$

where the map from $\mathbb{Z} / k p^{M} \mathbb{Z}$ to $\mathbb{Z} / k p^{N} \mathbb{Z}$ for $M \geq N$ is the reduction modulo $k p^{N}$. Denote by $a+k p^{N} \mathbb{Z}_{p}$ the set of $x$ in $X_{k}$ which map to $a$ in $\mathbb{Z} / k p^{N} \mathbb{Z}$ under the natural projection map from $X_{k}$ to $\mathbb{Z} / k p^{N} \mathbb{Z}$.

Fix a $r$-th root of unity $\varepsilon$ with $r$ relative prime to $k$. Also suppose that $\varepsilon$ is not a $p^{N}$-th root of unity for any $N$. Define

$$
\mu_{\varepsilon}\left(a+k p^{N} \mathbb{Z}_{p}\right)=\frac{\varepsilon^{a}}{1-\varepsilon^{k p^{N}}}
$$

and

$$
\mu\left(a+k p^{N} \mathbb{Z}_{p}\right)=\sum_{\substack{\varepsilon^{r}=1 \\ \varepsilon \neq 1}} \mu_{\varepsilon}\left(a+k p^{N} \mathbb{Z}_{p}\right)
$$


The above $p$-adic measure was given in [6] and it is also known as Mazure measure.

Note that

$$
X_{k}=\bigcup_{0 \leq a<k}\left(a+k \mathbb{Z}_{p}\right)
$$

is a disjoint union of $k$ topological spaces isomorphic to $\mathbb{Z}_{p}$. Also we have

$$
a+k p^{N} \mathbb{Z}_{p}=\bigcup_{0 \leq b<p}\left(\left(a+b k p^{N}\right)+k p^{N+1} \mathbb{Z}_{p}\right) .
$$

The above is a disjoint union of $p$ compact open sets. It is easy to verify directly that

$$
\mu\left(a+k p^{N} \mathbb{Z}\right)=\sum_{b=0}^{p-1} \mu\left(\left(a+b k p^{N}\right)+k p^{N+1} \mathbb{Z}_{p}\right) .
$$

For any continuous function $f: X_{k} \longrightarrow \Omega_{p}$, we define

$$
\int_{X_{k}} f(x) d \mu(x)=\lim _{N \longrightarrow \infty} \sum_{0 \leq a<k p^{N}} f(a) \mu\left(a+k p^{N} \mathbb{Z}_{p}\right) .
$$

Consider the integration of the exponential function $e^{t x}$ and follow the general procedure of [6], we obtain the following.

Proposition 16. For any positive integers $m$ and $k$, we have

$$
\int_{X_{k}} x^{m-1} d \mu(x)=\left(1-r^{m}\right) \frac{B_{m}}{m} .
$$

Proposition 17. Let $X_{k}^{*}$ be elements of $X_{k}$ which map onto $(\mathbb{Z} / k \mathbb{Z})^{*}$, the invertible elements of $\mathbb{Z} / k \mathbb{Z}$. Then for any positive integer $m$,

$$
\int_{X_{k}^{*}} x^{m-1} d \mu(x)=\left(1-r^{m}\right) \frac{B_{m}}{m} \prod_{p \mid k}\left(1-p^{m-1}\right) .
$$

Proof. By the inclusion-exclusion principle, we decompose the integration into the following:

$$
\int_{X_{k}^{*}}=\int_{X_{k}}-\sum_{p_{i} \mid k} \int_{p_{i} X_{k}}+\sum_{p_{i} p_{j} \mid k} \int_{p_{i} p_{j} X_{k}}+\cdots+(-1)^{\mu} \int_{p_{1} \cdots p_{\mu} X_{k}} .
$$


Here $p_{1}, \ldots, p_{\mu}$ are distinct prime divisors of $k$. To prove the proposition, it suffices to prove that

$$
\int_{\alpha X_{k}} x^{m-1} d \mu(x)=\left(1-r^{m}\right) \alpha^{m-1} \frac{B_{m}}{m},
$$

for any integer $\alpha$ which is a prime divisor or a product of distinct prime divisors of $k$.

Again we consider the integration of $e^{t x}$,

$$
\begin{aligned}
\int_{\alpha X_{k}} e^{t x} d \mu_{\varepsilon}(x) & =\lim _{N \rightarrow \infty}\left(1-\varepsilon^{k p^{N}}\right)^{-1} \sum_{0 \leq b<k p^{N} / \alpha}\left(\varepsilon e^{t}\right)^{\alpha b} \\
& =\lim _{N \rightarrow \infty}\left(1-\varepsilon^{k p^{N}}\right)^{-1}\left(1-\left(\varepsilon e^{t}\right)^{k p^{N}}\right)\left(1-\varepsilon^{\alpha} e^{\alpha t}\right)^{-1} \\
& =\left(1-\varepsilon^{\alpha} e^{\alpha t}\right)^{-1} .
\end{aligned}
$$

Since $r$ is relative prime to $\alpha$, the mapping $\varepsilon$ to $\varepsilon^{\alpha}$ causes a permutation among $r$-th roots of unity. Hence

$$
\begin{aligned}
\int_{\alpha X_{k}} e^{t x} d \mu(x) & =\frac{r-\left(1+e^{\alpha t}+\cdots+e^{(r-1) \alpha t}\right)}{1-e^{r \alpha t}} \\
& =\frac{r}{1-e^{r \alpha t}}-\frac{1}{1-e^{\alpha t}} \\
& =\sum_{m=1}^{\infty} \frac{\left(1-r^{m}\right) B_{m}(\alpha t)^{m-1}}{m !} .
\end{aligned}
$$

By comparing the coefficients of $t$, we get our assertion.

Now we are ready to given another proof of the theorem in Section 9.

Proof of Theorem 15 . For any element $x$ in $(\mathbb{Z} / k \mathbb{Z})^{*}$, we have the congruence relation

$$
x^{m-1} \equiv x^{n-1} \quad(\bmod k),
$$

since $m-n$ is a multiple of $\varphi(k)$. Hence for any prime divisor $p$ of $k$, with the $p$-adic measure $\mu(x)$ defined on $X_{k}$, we have

$$
\int_{X_{k}^{*}} x^{m-1} d \mu(x) \equiv \int_{X_{k}^{*}} x^{n-1} d \mu(x) \quad\left(\bmod p^{\alpha}\right),
$$


where $\alpha=\nu_{p}(k)$ is the highest power of $p$ dividing $k$. On the other hand, we have $r^{m}-1 \in(\mathbb{Z} / k \mathbb{Z})^{*}$ since $r^{m}-1 \in\left(\mathbb{Z} / p^{\alpha} \mathbb{Z}\right)^{*}$ for any prime divisor $p$ of $k$. Also we have

$$
r^{m}-1 \equiv r^{n}-1 \quad(\bmod k)
$$

since $(r, n)=1$ and $m \equiv n(\bmod \varphi(k))$. Hence

$$
\left(1-r^{m}\right)^{-1} \int_{X_{k}^{*}} x^{m-1} d \mu(x) \equiv\left(1-r^{n}\right)^{-1} \int_{X_{k}^{*}} x^{n-1} d \mu(x) \quad\left(\bmod p^{\alpha}\right) .
$$

This is equivalent to

$$
\frac{B_{m}}{m} \prod_{p \mid k}\left(1-p^{m-1}\right) \equiv \frac{B_{n}}{n} \prod_{p \mid k}\left(1-p^{n-1}\right) \quad\left(\bmod p^{\alpha}\right) .
$$

Thus it follows

$$
\frac{B_{m}}{m} \prod_{p \mid k}\left(1-p^{m-1}\right) \equiv \frac{B_{n}}{n} \prod_{p \mid k}\left(1-p^{n-1}\right) \quad(\bmod k) .
$$

\section{Congruences among Bernoulli polynomials.}

We are able to apply our previous arguments in Section 6 to derive congruences among Bernoulli polynomials or in general, the special values at negative integers of zeta functions associated with rational functions as considered before. Here we give a simple example to illustrate the general procedure.

Proposition 18. For a fixed prime odd number $p(p \geq 5)$ and any positive integer $k$ relative prime to $p$. Suppose that $\alpha, \beta$ are positive integers such that $1 \leq \alpha, \beta<k$ and $\alpha+j_{0} n=\beta p$ for some positive integer $j_{0}$ with $1 \leq j_{0} \leq p-1$. Then for all complex number $s$ with Res $>1$,

$$
\zeta\left(s ; \frac{\alpha}{k}\right)-p^{-s} \zeta\left(s ; \frac{\beta}{k}\right)=\left(p^{N+1}\right)^{-s} \sum_{\substack{1 \leq j \leq k p^{N+1} \\(j, p)=1 \\ j \equiv \alpha(\bmod k)}} \zeta\left(s ; \frac{j}{k p^{N+1}}\right) .
$$


Proof. Consider the zeta function $Z_{F}(s)$ associated with the rational function

$$
F(T)=\frac{T^{\alpha}}{1-T^{k}} .
$$

Obviously, we have

$$
Z_{F}(s)=k^{-s} \zeta\left(s ; \frac{\alpha}{k}\right) .
$$

Also from the identity

$$
F(T)=\frac{T^{\alpha}\left(1+T^{k}+\cdots+T^{(p-1) k}\right)}{1-T^{k p}},
$$

we conclude that

$$
\begin{aligned}
Z_{F}(s) & =(k p)^{-s} \sum_{j=0}^{p-1} \zeta\left(s ; \frac{\alpha+j k}{k p}\right) \\
& =(k p)^{-s} \zeta\left(s ; \frac{\beta}{k}\right)+(k p)^{-s} \sum_{\substack{j=0 \\
j \neq j_{0}}}^{p-1} \zeta\left(s ; \frac{\alpha+j k}{k p}\right) .
\end{aligned}
$$

On the other hand, we also have

$$
F(T)=\frac{T^{\alpha}\left(1+T^{k}+\cdots+T^{(p-1) k}\right)\left(1+T^{k p}+\cdots+T^{k p\left(p^{N}-1\right)}\right)}{1-T^{k p^{N+1}}} .
$$

Thus it follows also that

$$
\begin{aligned}
k^{-s} \zeta\left(s ; \frac{\alpha}{k}\right)-(k p)^{-s} \zeta\left(s ; \frac{\beta}{k}\right) & =(k p)^{-s} \sum_{\substack{j=0 \\
j \neq j_{0}}}^{p-1} \zeta\left(s ; \frac{\alpha+j k}{k p}\right) \\
& =\left(k p^{N+1}\right)^{-s} \sum_{\substack{1 \leq j<k p^{N+1} \\
(j, p)=1 \\
j \equiv \alpha(\bmod k)}} \zeta\left(s ; \frac{j}{k p^{N+1}}\right) .
\end{aligned}
$$

Multiply the factor $k^{-s}$ on both sides, we get our assertion. 
To simplify notation we write

$$
\sum_{j \equiv \alpha(\bmod k)}
$$

for

$$
\sum_{\substack{1 \leq j<k p^{N+1} \\(j, p)=1 \\ j \equiv \alpha(\bmod k)}}
$$

Proposition 19. Under the assumptions of the previous proposition and suppose that $m, n$ are positive integers such that $p-1$ is not a divisor of $m$. Then

$$
\begin{aligned}
\frac{1}{m}\left(B_{m}\left(\frac{\alpha}{k}\right)-p^{m-1}\right. & \left.B_{m}\left(\frac{\beta}{k}\right)\right) \\
& \equiv \frac{1}{n}\left(B_{n}\left(\frac{\alpha}{k}\right)-p^{n-1} B_{m}\left(\frac{\beta}{k}\right)\right)\left(\bmod p^{N+1}\right),
\end{aligned}
$$

if $m \equiv n\left(\bmod (p-1) p^{N}\right)$.

Proof. Set $s=1-m$ in the identity of Proposition 7, we get

$$
\begin{aligned}
\frac{1}{m}\left(B_{m}\left(\frac{\alpha}{k}\right)-\right. & \left.p^{m-1} B_{m}\left(\frac{\beta}{k}\right)\right) \\
& =\frac{1}{m} \sum_{j \equiv \alpha(\bmod k)} \sum_{l=0}^{m}\left(\begin{array}{c}
m \\
l
\end{array}\right) B_{l} j^{m-l} p^{(N+1)(l-1)} k^{l-m} .
\end{aligned}
$$

With exact the same argument as in Proposition 2, we get

$$
\begin{aligned}
& \frac{1}{m}\left(B_{m}\left(\frac{\alpha}{k}\right)-p^{m-1} B_{m}\left(\frac{\beta}{k}\right)\right) \\
& \equiv \frac{1}{m k^{m} p^{N+1}} \sum_{j \equiv \alpha(\bmod k)} j^{m}-\frac{1}{2} \sum_{j \equiv \alpha(\bmod k)} j^{m-1} k^{1-m}\left(\bmod p^{N+1}\right) .
\end{aligned}
$$

Thus our congruences are equivalent to

$$
\frac{1}{m k^{m} p^{N+1}} \sum_{j \equiv \alpha(\bmod k)} j^{m} \equiv \frac{1}{n k^{n} p^{N+1}} \sum_{j \equiv \alpha(\bmod k)} j^{n} \quad\left(\bmod p^{N+1}\right) .
$$


Note that $k$ is relative prime to $p$, so the mapping $x \longmapsto k x+\alpha$ is an one to one mapping from $\mathbb{Z} / p^{N+1} \mathbb{Z}$ into $\mathbb{Z} / p^{N+1} \mathbb{Z}$. Thus we have

$$
\sum_{j \equiv \alpha(\bmod k)} j^{m} \equiv \sum_{\substack{\left.1 \leq j<p^{N+1} \\ j, p\right)=1}} j^{m}\left(\bmod p^{N+1}\right) .
$$

Hence our congruences follow by the same argument as in Proposition 13.

REMARK. It is possible to construct another $p$-adic measure on the space $\mathbb{Z}_{p}$ so that the integration of the monomial $x^{m-1}$ over $\mathbb{Z}_{p}^{*}$ yields a sum of Bernoulli polynomials. Hence, we have the $p$-adic interpolation of Kummer's congruences on Bernoulli polynomials. We'll discuss this in another paper.

\section{References.}

[1] Berndt, B. C., Ramanujan's Notebooks, I, II. Springer-Verlag, 1985, 1989.

[2] Borevich, Z. I., Shafarevich, I. R., Number Theory. Academic Press, 1996.

[3] Eie, M., The special values at negative integers of Dirichlet series associated with polynomials of several variables. Proc. Amer. Math. Soc. 119 (1993), 51-61.

[4] Eie, M., A note on Bernoulli numbers and Shintani generalized Bernoulli polynomials. Trans. Amer. Math. Soc. 348 (1996), 1117-1136.

[5] Eie, M., Dimension formula for vector spaces of Siegel cusp forms of degree three. Mem. Amer. Math. Soc. 373 (1987), 1-124.

[6] Hardy, G. H., Wright, E. M., An introduction to the theory of numbers. Oxford University Press, 1954.

[7] Igusa, J.-I., On Siegel modular forms of genus 2. Amer. J. Math. 84 (1962), 175-200.

[8] Iwasawa, K., Lecture on p-adic L-functions. Princeton University Press, 1972.

[9] Koblitz, N., p-adic analysis: a short course on recent work. Cambridge University Press, 1980.

[10] Rademacher, H., Topics in analytic number theory. Springer-Verlag, 1971.

[11] Rotman, J. J., Theory of Groups. Allyn and Bacon, 1965. 
[12] Serre, J. P., A course in arithmetic. Springer-Verlag, 1985.

[13] Shintani, T., On evaluation of zeta functions of totally real algebraic number fields at non-positive integers. J. Fac. Sci. Univ. Tokyo, Sect. IA, Math. 23 (1976), 393-417.

[14] Washington, L. C., Introduction to cyclotomic fields. Springer-Verlag, 1982.

[15] Zagier, D., Valeurs des fountions zêta des corps quadratiques réels aux entiers négatifs. Astérisque 41-42 (1977), 393-417.

[16] Zagier, D., Special values and functional equations of polylogarithms. In L. Lewin, ed., Structural properties of polylogarithms. Amer. Math. Soc. Math. Surveys \& Monographs 37 (1991), 377-400.

Recibido: 3 de marzo de 1.997

Minking Eie*

Department of Mathematics

National Chung Cheng

University Ming-Shiung

Chia-Yi, TAIWAN

mkeie@math.ccu.edu.tw

and

King F. Lai

School of Mathematics and Statistics

University of Sydney

NSW 2006, AUSTRALIA

kflai@maths.su.oz.au

* This work was supported by the Department of Mathematics, National Chung Cheng University and National Science Fundation of Taiwan, Republic of China 